\title{
Characterizing block-copolymer micelles used in nanomedicines via solution static scattering techniques
}

\author{
Isamu Akiba $\mathbb{D}^{1} \cdot$ Kazuo Sakurai ${ }^{1}$
}

Received: 31 July 2020 / Revised: 11 December 2020 / Accepted: 13 December 2020 / Published online: 31 May 2021

(c) The Author(s) 2021. This article is published with open access

\begin{abstract}
Block copolymers are well recognized as excellent nanotools for delivering hydrophobic drugs. The formulation of such delivery nanoparticles requires robust characterization and clarification of the critical quality attributes correlating with the safety and efficacy of the drug before applying to regulatory authorities for approval. Static solution scattering from block copolymers is one such technique. This paper first outlines the theoretical background and current models for analyzing this scattering and then presents an overview of our recent studies on block copolymers.
\end{abstract}

\section{Introduction}

Amphipathic blockcopolymers in aqueous solutions may undergo microphase separation to form micelles, a consequence of chemically different blocks repelling each other and eventually segregating into two domains in accordance with the level of hydrophobicity. The hydrophilic domain contains a large amount of water and covers the hydrophobic domain, which decreases the interfacial free energy arising from contact with water. By changing the chainlength ratio between the two blocks, their morphology can be controlled. Some amphipathic blockcopolymers show quite rich and strange morphologies [1-3], probably because them are kinetically frozen in most cases. Among the others, stable spherical micelles consisting of a hydrophobic core and a hydrophilic shell can be obtained, as illustrated in Fig. 1. In most cases, the hydrophilic block length is approximately the same or longer than the hydrophobic block length (i.e., $L_{\mathrm{S}} \geq L_{\mathrm{C}}$; here, $L_{\mathrm{S}}$ and $L_{\mathrm{C}}$ are the chain length or degree of polymerization of the shell and core chains, respectively). The most fundamental structural parameters for describing such spherical micelles may be the average aggregation number $\left(N_{\mathrm{agg}}\right)$ and the core and shell sizes, defined by the radii of the core $\left(R_{\mathrm{C}}\right)$ and shell

Kazuo Sakurai

sakurai@kitakyu-u.ac.jp

1 Department of Chemistry and Biochemistry, The University of Kitakyushu, Fukuoka, Japan
$\left(R_{\mathrm{S}}\right)$, as shown in Fig. 1. Unlike oil droplets, the spherical micelles composed of blockcopolymers do not grow to the macroscopic scale. In fact, the size is defined by a characteristic spatial length scale such as the correlation length, which is why the term microphase separation is used. The characteristic length is governed mainly by the chain lengths, but more accurately by the thermodynamic balance among several factors as mentioned below. The size distribution is normally quite narrow when the original block copolymers have a reasonably narrow range of molecular weights. The micellar properties of amphipathic block copolymers in aqueous solutions have been reviewed by several groups $[4,5]$, including an early study by Tuzar and Kratochvil [6]. Among the others, an excellent book comprehensively reviewing block copolymers as well as their dilute solution properties for findings reported up to 2005 was published by Hamley $[5,7]$.

Polymeric micelles are expected to play an important role in the drug delivery system (DDS) for poorly water-soluble drugs and therapeutic oligonucleotides [8-12]. The hydrophilic shell reduces opsonin adsorption on the particle and enables the evasion of immune recognition by various immunocytes in the blood, which leads to a long blood circulation time. This evasion of immune recognition is called the "stealth effect". [13] The core can contain a drug through hydrophobic or electrostatic interactions between them, as shown in Fig. 1. When the particle size is adjusted to within a suitable range [14], normally $10-100 \mathrm{~nm}$, the particles show a longer blood circulation time by evading clearance by mononuclear phagocytes in the liver and bypassing filtration in the kidney. Ultimately, a longer 


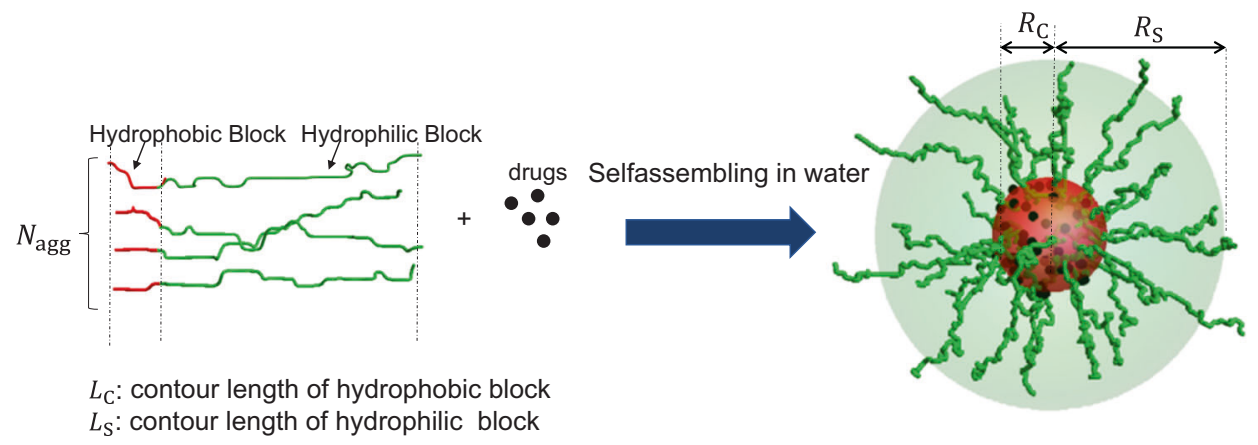

Fig. 1 A schematic illustration of the self-assembly of amphipathic block copolymers in aqueous solution to form a spherical micelle. When hydrophobic drugs are present in this process, they may be automatically incorporated into the micellar hydrophobic core by

circulation time leads to accumulation of the drug at tissue sites with vascular abnormalities such as tumors. Once the particles enter the tumor region after penetrating the endothelial barrier, they may further penetrate the tumor by diffusion or hydrophobic interaction with the cellular membrane. Since there is no lymphatic clearance in a tumor, the accumulated particles are retained there. This overall phenomenon is called the enhanced permeation and retention effect (EPR effect) $[15,16]$, which is the basic route of entry of all nanoscale DDSs developed for antitumor therapy. Several comprehensive reviews of the EPR effect have recently been published $[8,10,17]$.

Theoretical predictions for the block length dependence of structural parameters

The most important factor in determining the micellar structures is the volumetric balance between the hydrophilic and hydrophobic groups, which is well known as the packing parameter principle $[18,19]$. For the core-volume $\left(V_{\mathrm{C}}\right)$ and the interfacial surface area $\left(A_{\mathrm{C}}\right)$ of a sphere made up of $N_{\mathrm{agg}}$ molecules, $R_{\mathrm{C}}$ cannot exceed the extended chain length $h$ of the core-block, that is., $R_{\mathrm{C}}<h$, owing to the geometric constraint. For spherical micelles, $A_{\mathrm{C}}=a_{\mathrm{C}} N_{\mathrm{agg}}=4 \pi\left(R_{\mathrm{C}}\right)^{2}$ and $V_{\mathrm{C}}=v_{\mathrm{C}} N_{\mathrm{agg}}$, where $a_{\mathrm{C}}$ and $v_{\mathrm{C}}$ are the interfacial area and the core volume per block, respectively. $V_{\mathrm{C}} / A_{\mathrm{C}}=R_{\mathrm{C}} / 3$ and $V_{\mathrm{C}} / A_{\mathrm{C}}=v_{\mathrm{C}} / a_{\mathrm{C}}$. Therefore, $v_{\mathrm{C}} /\left(a_{\mathrm{C}} h\right)<1 / 3$. To form spherical micelles, the parameter $v_{\mathrm{C}} /\left(a_{\mathrm{C}} h\right)$ must be less than $1 / 3$. This dimensionless index is well known as the packing parameter $[18,19]$. To understand equilibrium structures in more detail, we may have to consider the following factors: (1) the entropic elasticity of the core chains; (2) the free energy change due to solubilization of small molecules (solvent or drug) into the core; (3) the free energy of the shell chains given by the combination of the elastic stretching of the shell chains, the excluded volume effect between the chain segments, and the osmotic chemical potential due to solvent; and (4) the interfacial tension arising from unfavorable contact between the hydrophobic core chains and water molecules. means of hydrophobic interactions. Some of the important physical parameters are schematically illustrated; $N_{\text {agg }}$ aggregation number, $L_{\mathrm{C}}$ contour length of the hydrophobic core chain, $L_{\mathrm{S}}$ contour length of the hydrophilic shell chain, $R_{\mathrm{S}}$ radius of core, and $R_{\mathrm{S}}$ radius of shell

A simple scaling model of block-copolymer micelles was proposed by Gennes [20, 21], on the assumption that the core chains are completely segregated from both the shell chains and solvent molecules, forming a melt or solid-like core, and the shell chain and solvent molecules are uniformly mixed in the shell region. In this model, the elastic deformation of the core chain, that is, conformational entropy, plays a major role in determining $N_{\text {agg }}$ and this theory predicts:

$$
N_{\text {agg }} \propto L_{C}
$$

here, the core size is related to $N_{\text {agg }}$ through $R_{\mathrm{C}} \propto \sqrt[3]{N_{\mathrm{agg}} L_{\mathrm{C}}}$. This model assumes a constant chain concentration in the shell domain, considering that the shell chains have no influence on the micellar characteristics and thus the aggregation size.

By analogy with the overlap concentration $\left(\mathrm{c}^{*}\right)$, which is the boundary between the dilute and semidilute regimes of polymer solutions, the crowding of the shell chains can be classified into two regions: (a) isolated and (c) overcrowded as illustrated in Fig. 2. The overlap state (b) corresponds to $\mathrm{c}^{*}$. More precisely, the isolated state is defined by $\sigma<1$ by using the surface coverage index $\sigma$ given by the following Eq. [22].

$$
\sigma=\frac{\pi R_{\mathrm{g}, \mathrm{Sh}}^{2} N_{\mathrm{agg}}}{4 \pi\left(R_{\mathrm{C}}+R_{\mathrm{g}, \mathrm{Sh}}\right)^{2}}
$$

here, $R_{\mathrm{g}, \mathrm{Sh}}$ is the radius of gyration of the shell chain. The de Gennes model may be valid for only (a) and (b) states. In the (c) state, the shell chain-segment density may be higher at the core-shell interface and become lower closer to the water-shell interface [23]. This tendency should be more pronounced in the case of $L_{\mathrm{S}}>L_{\mathrm{C}}$ or large $N_{\mathrm{agg}}$. Zhulina and Birshtein [24], and Halperin [23] used the star-polymer model formulated by Daoud and Cotton [25] to describe 


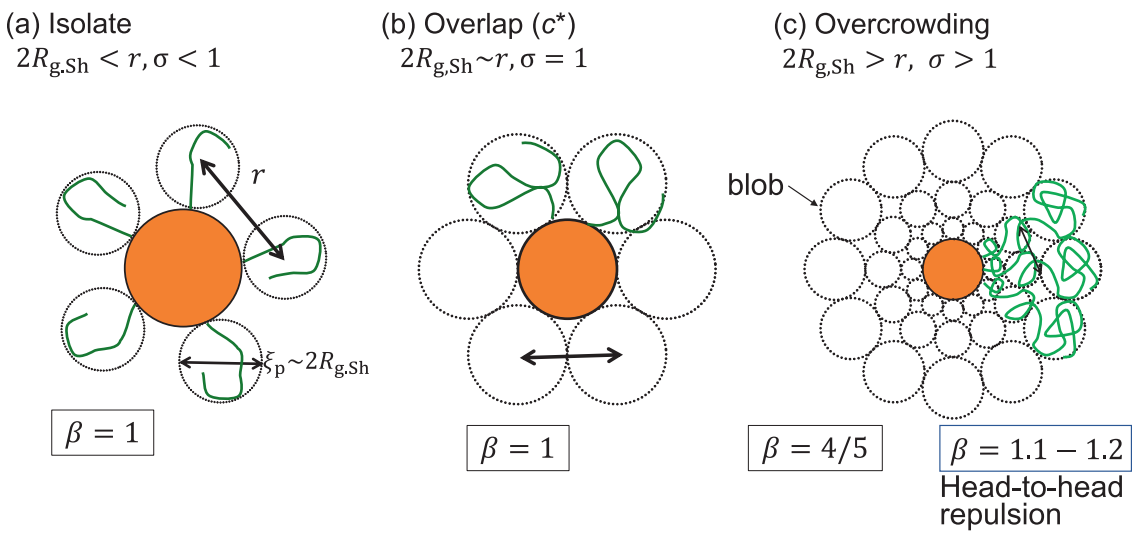

Fig. 2 Cross-sectional drawings of polymer micelles for the two distinctively different states for the shell chain crowding. a The shell chains are isolated far enough away so as not to interact with each other; the neighboring distance $(r)$ is larger than twice the radius of gyration $\left(R_{\mathrm{g}, \mathrm{Sh}}\right)$ and the surface coverage index $(\sigma)$ given by Eq. 2 is

such a density gradation of the shell chains. Their model leads to the following relation:

$N_{\text {agg }} \propto L_{\mathrm{C}}^{4 / 5}$

It is interesting that $N_{\text {agg }}$ is still independent of $L_{\mathrm{S}}$ and the scaling exponent of $L_{\mathrm{C}}$ becomes smaller than that of de Gennes' model. Nagarajan and Ganesh [26] improved this model by incorporating the solubilization interaction between solvents and the shell chains into the Daoud and Cotton model [26]. Their results showed a weak influence from $L_{S}$ through logarithmic dependence. In low-molecularweight surfactants, the repulsive interaction between the hydrophilic head groups is one of the major factors determining the aggregation number. Nagarajan and Ganesh considered that an analogous repulsive interaction between the shell chains should be important in polymeric micelles. However, the star-polymer models do not account for this factor. Nagarajan et al. [27-29] introduced the osmotic and elastic contributions into the Daoud and Cotton model and found the following relation for a good solvent of the shell chains:

$N_{\mathrm{agg}} \propto\left(L_{\mathrm{C}}\right)^{1.19}\left(L_{\mathrm{S}}\right)^{-0.87}$

This relation suggests that the core block gives a stronger dependence on $N_{\text {agg }}$ than the previous models, indicating that the repulsions (or osmotic contribution) of the shell chains are very important for block copolymers when the selective solvent is a very good solvent for the shell chains. By combining numerical results for experimental data, Nagarajan et al. obtained a "universal relation" for $N_{\text {agg }}$ as well as the core and micellar sizes [5, 7, 27]. In the past three decades, there have been many studies examining the less than 1. b The crowding of the shell chains corresponds to the overlap concentration $\left(\mathrm{c}^{*}\right)$. c The shell chains are so crowded that $2 R_{g}>r$ and $\sigma>1 . \beta$ is the scaling exponent in the case of expressing $N_{\text {agg }} \propto L_{C}^{\beta}$

theoretical predictions on this issue of block-copolymer micelles in organic and aqueous solutions [30-37].

\section{Importance of accurate characterization from the regulatory science perspective}

The development of new drugs must be carried out under the strictest governmental regulations. From drug discovery to registration, pharmaceutical companies and other drug developers are required to negotiate with the regulatory authorities through complex and lengthy processes. These processes are necessary to ensure that new drugs are safe and effective and were established after several appalling adverse drug events [38]. The development of a single drug is estimated to take 15-25 years to progress from initial research to final commercialization, and this development cost more than 1 billion US dollars in the early 2000s. The major developmental stages from drug discovery to marketing are presented in Fig. 3. The lead compound identified in the drug-candidate discovery process is examined in vitro and in vivo to check how the compound affects biological systems, including toxicology, pharmacodynamics, pharmacokinetics, and optimization of its formulation. It is essential to establish the relationship between its formulation/process variables and critical quality attributes (CQAs) for safety and efficacy; this is called "quality by design". All of the data should be recorded so that any third party can reconstruct the study without assumptions or preconceptions. The investigator submits an investigational new drug (IND) to the Food and Drug Administration (FDA) in the US or to similar organizations in other countries. Once the FDA has approved the IND, the drug candidate will be evaluated in a first-in-human or phase I clinical trial, 
Fig. 3 Drug development process from discovery of drug candidate to FDA approval and the increase in the number of nanomedicine application to CDER per year. Applications are separated into IND (Investigational New Drug), NDA (New Drug Application), and ANDA (Abbreviated New Drug Application). GLP, GCP, and cGMP means "Good Laboratory Practice", "Good Clinical Practice", and "Current Good Manufacturing Practice", respectively

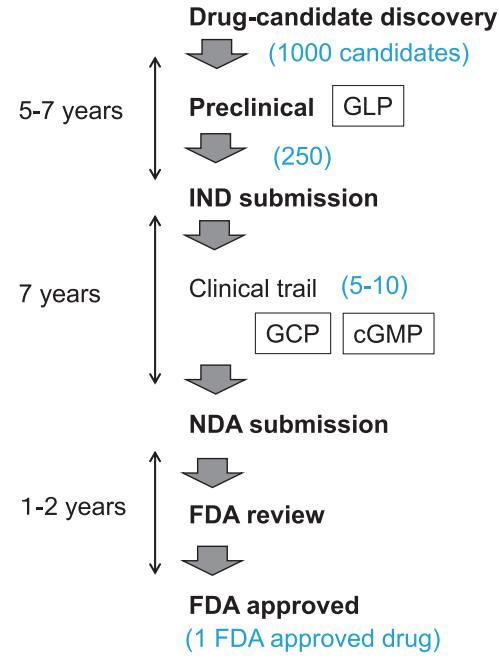

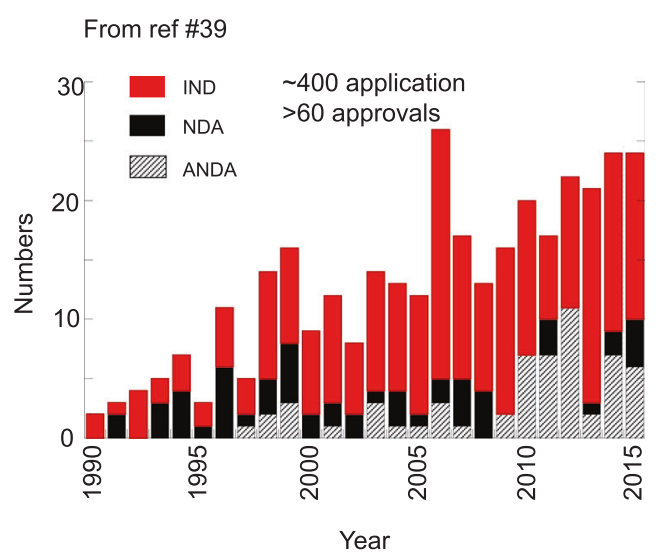

followed by phase II and III trials, and finally submitted for a new drug application (NDA). As shown in Fig. 3, the whole process takes 15-20 years and the FDA approval rate is $\sim 0.1 \%$ or less.

According to the Center for Drug Evaluation and Research (CDER) at the FDA [39], which evaluates new drug applications, there has been a substantial increase in the submissions of drug products containing nanomaterials over the last two decades, as shown in Fig. 3. Approximately $80 \%$ of products have average particle sizes of $300 \mathrm{~nm}$ or lower and are considered nanomedicines [40]. When compared with the number of "classical" small-molecule drugs, clinically approved nanomedicines are still very limited [39, 41]. Despite the large number of nanomedicines reported in the literature, only $\sim 50$ candidates have been successfully translated to clinical use. Nanomedicines often fail to reach late clinical phases due to the lack of preclinical characterization protocols that satisfy the regulatory authorities [41, 42]. Compared with the case for small-molecule drugs, the safety and efficacy profiles of nanomedicines demand the characterization of many additional physicochemical properties, including average particle size and polydispersity, dispersion stability, particle shape, surface charge, drug loading and drug release, surface coating, and hydrophobicity [42, 43]. In this context, the US National Cancer Institute Nanotechnology Characterization Laboratory (NCI-NCL) and the European Nanomedicine Characterization Laboratory (EUNCL) have established standard operating procedures (SOPs) for nanomedicines, which are published online (http://www.euncl.eu/a bout-us/assay-cascade/). It is generally believed that the particle size, shape, charge, surface area, and drug-release rate of nanomedicines may change the pharmacokinetics, biodistribution, and toxicity, thus affecting the therapeutic effect. In other words, these are CQAs of nanomedicines.

Polymeric micelles are quite new from drug formulation and regulatory perspectives [17]. However, new quality control assays and robust techniques for characterizing polymeric micelles are now widely acknowledged to be needed [44]. In 2013, an important document, "Joint MHLW/EMA reflection paper on the development of blockcopolymer micelle medicinal products", was released [45]. According to this document, the following 11 items are considered as the properties relevant for characterizing the quality of the finished product for polymeric micelles: (1) micelle size (mean and distribution profile), (2) morphology (i.e., structural characterization), (3) zeta potential, (4) aggregation number, (5) concentration dependence of the nanostructure (critical micelle concentration or critical association concentration), (6) drug loading, (7) physical state of the active substance, (8) viscosity, (9) in vitro stability of the block-copolymer micelle in plasma and/or relevant media, (10) in vitro release of the active substance from the blockcopolymer micelle product in plasma and/or relevant media, and (11) in vitro degradation of the block copolymer in plasma and/or relevant media. Some of these are in common with the liposome draft guidance published by CDER [46].

Among these items, the particle size, the total molecular weight of the particle, and their distribution are the most important and essential CQAs. A recent analysis based on the applications submitted to the FDA from 1973 to 2015 showed that dynamic light scattering (DLS) has been most commonly used for the determination of particle size (48\%), followed by static laser or light scattering $(30 \%)$ and electron or optical microscopy (14\%) [39]. Only $8 \%$ or fewer applicants used static scattering coupled with a fractionation technique such as gel permeation chromatography or symmetrical flow fieldflow fractionation (FFF). Despite being commonly used to characterize nanomedicines, several assumptions need to be made to use DLS to obtain their size, so it is generally considered a "low-resolution" method [42, 43, 47]. DLS provides only the diameter of a solid sphere hydrodynamically equivalent to the measured particle and a valid distribution 
only for monodisperse systems, which is now clearly recognized by the regulatory agencies. Despite requiring more complicated procedures and expertise, static scattering, especially X-ray small-angle scattering, can provide detailed and accurate structures of the particles in solution. In the next section, the basic principles of both static light scattering and $\mathrm{X}$-ray scattering are summarized.

\section{Theoretical background of static scattering}

\section{General formula}

The absolute scattering intensity of spherical wave $\left[I_{\mathrm{S}}(q)\right]$ from the scattering volume $V$ which contains $N$ randomly oriented objects in solution, may be expressed as a function of the magnitude of the scattering vector $|\mathbf{q}| \equiv q=(4 \pi / \lambda) \sin$ $\theta / 2$; here, $\lambda$ and $\theta$ are the wavelength of the incident light or $\mathrm{X}$-ray, and the scattering angle, respectively.

$I_{\mathrm{S}}(q)=\frac{I_{\mathrm{i}}}{R_{\mathrm{D}}^{2}}\left\langle\left|F_{\mathrm{S}}(\mathbf{q})\right|^{2}\right\rangle_{\mathbf{q}}$

here $I_{\mathrm{i}}$ and $R_{\mathrm{D}}$ are the intensity of the incident beam and the distance between the sample and the detector, respectively. $\left|F_{\mathrm{S}}(\mathbf{q})\right|^{2}$ is the structural factor of the scattering object and $\left|F_{\mathrm{S}}(\mathbf{q})\right|^{2} / V$ equals the differential scattering cross section: the ratio of the scattered flux (or number of particles) in a given direction per unit time per unit solid angle to the incident flux. The bracketed \langle\rangle$_{\mathbf{q}}$ indicates the rotational average in the q-space. By subtracting the scattering intensity of the solvent from that of the solution, the value of $\left\langle\left|F_{\mathrm{S}}(\mathbf{q})\right|^{2}\right\rangle_{\mathbf{q}} / V$ can be ascribed to only solutes, which is called the excess scattering intensity, named the Rayleigh ratio $\left[R_{\theta}(q)\right]$ in light scattering and simply called the scattering intensity $[I(q)]$ in small-angle X-ray scattering. The structural factor is related to the scattering lengths ${ }_{p i}$ and $p_{j}$ at points $i$ and $j$ in the scattering volume, and the vector $\mathbf{r}_{i j}$ defined by the difference of two position vectors, namely, $\mathbf{r}_{i j} \equiv \mathbf{r}_{i}-\mathbf{r}_{j}$

$$
\left|F_{\mathrm{S}}(\mathbf{q})\right|^{2}=\left|\sum_{i} p_{i} \exp \left(-i \mathbf{q} \cdot \mathbf{r}_{i}\right)\right|^{2}=\sum_{i} \sum_{j} p_{i} p_{j}^{*} \exp \left(-i \mathbf{q} \cdot \mathbf{r}_{i j}\right)
$$

The scattering length $p i$ is related to the scattering-length density $p(\mathbf{r})$ through $p=p(\mathbf{r}) \mathbb{d} \mathbf{r}$. For X-rays and light, $p(\mathbf{r})$ is related to the electron density $\rho_{\mathrm{e}}(\mathbf{r})$ through $p(\mathbf{r})=r_{\mathrm{e}} \rho_{\mathrm{e}}(\mathbf{r}) \sin \gamma$ and the polarizability density $\alpha_{\mathrm{m}}(\mathbf{r})$ by $p(\mathbf{r})=k_{\mathrm{S}, 0}^{2} \alpha_{\mathrm{m}}(\mathbf{r}) \sin \gamma$, respectively. Here, $r_{\mathrm{e}}$ and $k_{\mathrm{S}, 0}$ are the classical radius of an electron (i.e., $\left.r_{\mathrm{e}}=e^{2} /\left(m c^{2}\right)=2.818 \times 10^{-6} \mathrm{~nm}\right)$ and the wavenumbers of the incident light in vacuum (i.e., $k_{\mathrm{S}, 0}=2 \pi / \lambda_{0}$ ), respectively, and $\sin \gamma$ is related to the polarization of the incident beam and $\sin ^{2} \gamma=\left(1+\cos ^{2} \theta\right) / 2$ for the unpolarized beam. It should be noted that this difference due to the two different light source is ascribed to the difference between the Thomson and Rayleigh scatterings; in the former case, the $\mathrm{X}$-ray energy is much greater than the energy of the characteristic vibration of electrons, while in the latter case, the light energy is much smaller. This leads to the scattering length of light $p_{\text {light }} \propto \lambda_{0}{ }^{-2}$ and the scattering length of X-rays $p_{\mathrm{X}-\text { ray }}=$ constant. By using $p(\mathbf{r}) \mathbb{d} \mathbf{r}$, the summation of $\sum_{i p i} \exp \left(-i \mathbf{q} \cdot \mathbf{r}_{i}\right)$ can be transformed to an integral;

$$
\begin{aligned}
F_{\mathrm{S}}(\mathbf{q})= & \int p(\mathbf{r}) \exp (-i \mathbf{q} \cdot \mathbf{r}) \mathbb{d} \mathbf{r}=\int\left[p(\mathbf{r})-p_{\mathrm{sol}}\right] \\
& \exp (-i \mathbf{q} \cdot \mathbf{r}) \mathbb{d} \mathbf{r}+(2 \pi)^{3} p_{\mathrm{sol}} \delta(\mathbf{q})
\end{aligned}
$$

The last term is a delta function and is centered at $\mathbf{q}=0$, which is invisible for ordinal cases. Equation 7 indicates that the scattering length in Eq. 5 is replaced by $p_{i}-p_{\text {sol }}$, which is called the excess scattering length. When we consider scattering from $N$ particles, an ensemble average over different particles is necessary. By relabeling each particle with two subscripts $i$ and $k$, Eq. 6, where the $i$-th position in the $k$-th particle and $N$ and $n_{k}$ are the number of particles and the number of positions in the $k$-th particle, respectively, Eq. 6 leads to the following:

$$
\begin{aligned}
\left\langle\left|F_{\mathrm{S}}(\mathbf{q})\right|^{2}\right\rangle_{\mathrm{en}} & =\left\langle\left|\sum_{k=1}^{N} \sum_{i=1}^{n_{k}} p_{k i} \exp \left(-i \mathbf{q} \cdot \mathbf{r}_{k i}\right)\right|^{2}\right\rangle \\
& =\left\langle\left|\sum_{k=1}^{N} \exp \left(-i \mathbf{q} \cdot \mathbf{R}_{k}\right) \sum_{i=1}^{n_{k}} p_{k i} \exp \left(-i \mathbf{q} \cdot \mathbf{X}_{k i}\right)\right|^{2}\right\rangle
\end{aligned}
$$

The third term can be obtained, using the center of mass of the $k$-th particle and the position of the $i$-th at $\mathbf{r}_{\mathrm{ki}}$ relative to the mass center by $\mathbf{X}_{i}$, i.e., $\mathbf{r}_{k i}=\mathbf{R}_{k}+\mathbf{X}_{k i}$. Next, the structural amplitude of the k-th particle can be defined as $F_{k}(\mathbf{q})=\sum_{i}^{n_{k}} p_{k i} \exp \left(-i \mathbf{q} \cdot \mathbf{X}_{k i}\right)$, and then:

$$
\left\langle\left|F_{\mathrm{S}}(\mathbf{q})\right|^{2}\right\rangle_{\mathrm{en}}=\left\langle\sum_{k=1}^{N} \sum_{k^{\prime}=1}^{N} F_{k^{\prime}} *(\mathbf{q}) F_{k}(\mathbf{q}) \exp \left[-i \mathbf{q}\left(\mathbf{R}_{k}-\mathbf{R}_{k^{\prime}}\right)\right]\right\rangle_{\mathrm{en}}
$$

When we can assume that the particle size and orientation are not correlated with the particle position of $\mathbf{R}_{k}, F_{k}{ }^{*}(\mathbf{q}) F_{k}$ (q) can be ensemble-averaged over $k=0$ to $N$ independently of their position. This leads to $\left\langle F_{k^{\prime}}{ }^{*}(\mathbf{q}) F_{k}(\mathbf{q})\right\rangle_{\text {en }}=\mid\langle F$ (q) $\rangle\left._{\text {en }}\right|^{2}$ for $k \neq k^{\prime}$ or $=\left\langle|F(\mathbf{q})|^{2}\right\rangle_{\text {en }}$ for $k=k^{\prime}$. Then, the structural factor reduces to;

$$
\left\langle\left|F_{\mathrm{S}}(\mathbf{q})\right|^{2}\right\rangle_{\mathrm{en}}=N\left\langle|F(\mathbf{q})|^{2}\right\rangle_{\mathrm{en}}+N\left|\langle F(\mathbf{q})\rangle_{\mathrm{en}}\right|^{2} S(\mathbf{q})
$$


where $S(\mathbf{q})=\Sigma \boldsymbol{\Sigma}_{\mathrm{k \neq k} \mathrm{k}^{\prime}} \exp \left[-i \mathbf{q}\left(\mathbf{R}_{k}-\mathbf{R}_{k^{\prime}}\right)\right] / N$. It is clear from the definitions that $S(\mathbf{q})$ is related to only relative particle positions for different particles and is called the interparticle interference factor. $S^{\prime}(\mathbf{q})$ can then be defined as follows:

$S^{\prime}(\mathbf{q})=1+\frac{\left|\langle F(\mathbf{q})\rangle_{\text {en }}\right|^{2}}{\left\langle|F(\mathbf{q})|^{2}\right\rangle_{\text {en }}} S(\mathbf{q})$

The ensemble-averaged structural factor is written by;

$$
\left\langle\left|F_{S}(\mathbf{q})\right|^{2}\right\rangle_{\mathrm{en}}=N\left\langle|F(\mathbf{q})|^{2}\right\rangle_{\mathrm{en}} S^{\prime}(\mathbf{q})
$$

$\left\langle|F(\mathbf{q})|^{2}\right\rangle_{\text {en }}$ is called the form factor or intraparticle interference factor of the particles. When the scattering particles are polydisperse or non-centrosymmetric, $\left|\langle F(\mathbf{q})\rangle_{\mathrm{en}}\right|^{2} \neq\left\langle|F(\mathbf{q})|^{2}\right\rangle_{\mathrm{en}}$ and thus $S^{\prime}(\mathbf{q})$ contains the information of the particle shape. In the case of $\left|\langle F(\mathbf{q})\rangle_{\mathrm{en}}\right|^{2}=\left\langle|F(\mathbf{q})|^{2}\right\rangle_{\mathrm{en}}, S^{\prime}(\mathbf{q})=1+S(\mathbf{q})$ and thus the intra- and inter- particle interferences (i.e., $\left\langle|F(\mathbf{q})|^{2}\right\rangle$ and $S^{\prime}(\mathbf{q})$ ) are separated or decoupled; however, this is true only for monodisperse and centrosymmetric particles $[48,49]$. When the system can be regarded as being close enough to fulfill such conditions, called the decoupling approximation, $S(\mathbf{q})$ becomes a function of only $q$ after rotating-averaging over $\mathbf{q}$ :

$$
\begin{aligned}
\left\langle S^{\prime}(\mathbf{q})\right\rangle_{\text {decup }+\mathbf{q}} & =1+\langle S(\mathbf{q})\rangle_{\mathbf{q}} \equiv \mathcal{F}(q) \\
& =1-\frac{N}{V} \int_{0}^{\infty}[1-P(R)] \frac{\sin q R}{q R} 4 \pi R^{2} \mathbb{d} R
\end{aligned}
$$

here, $\mathcal{F}(q)$ is called the particle interference factor and $P(R)$ is the radial distribution function: the probability of finding the other particles in the finite shell of $4 \pi R^{2} \mathbb{d} R$ when one particle exists at the origin. When the number of particles is small enough, the particle position becomes random and uniform. In this case, $\lim _{N / V \rightarrow 0} \mathcal{F}(q)=1$ and $\left\langle\left|F_{\mathrm{S}}(\mathbf{q})\right|^{2}\right\rangle_{\mathrm{en}}=$ $N\left\langle|F(\mathbf{q})|^{2}\right\rangle$ (independent scattering). Experimentally speaking, the independent scattering condition can be achieved by extrapolating the concentration $(c)$ to zero. At this limit, the particle scattering function $P_{\theta}(q)$ can be defined as follows:

$P_{\theta}(q)=\frac{\lim _{c \rightarrow 0} I_{\mathrm{S}}(q)}{\lim _{c \rightarrow 0, q \rightarrow 0} I_{\mathrm{S}}(q)}=\frac{\left\langle|\mathrm{F}(\mathbf{q})|^{2}\right\rangle_{\mathrm{en}}}{\left\langle|\mathrm{F}(0)|^{2}\right\rangle_{\mathrm{en}}}$

Some papers show that $N\left\langle|F(\mathbf{q})|^{2}\right\rangle_{\text {en }} \mathcal{F}(q)$ was applied to isolate the form factor of the particles or the particle interference factor; furthermore, the interparticle potential function was derived from $P(R)$. However, such an analysis is valid only for monodisperse and centrosymmetric particles.
When the scattering objects have spherical symmetry in the scattering length distribution, that is, $p(\mathbf{r})=p(r), F(\mathbf{q})$ in Eq. 10 depends only on $q$ and can be expressed as follows:

$F(q)=\int_{0}^{\infty} p(r) \frac{\sin q r}{q r} 4 \pi r^{2} \mathbb{d} r$

At the limit of $q \rightarrow 0, F(0)=\int_{0}^{\infty} p(r) 4 \pi r^{2} \mathbb{d} r$, which can be expressed by $p_{\mathrm{av}} V_{\mathrm{S}}$, with the average excess scattering length $\left(p_{\text {av }}\right)$ multiplied by the volume of the scattering object $\left(V_{\mathrm{S}}\right)$. At small $q$ and under independent scattering conditions, since $\sin q r /(q r) \cong 1-(q r)^{2} / 6$, the excess ratio $R_{\theta}(q)$ or $I(q)$ from an $N$ particle system may be given by;

$$
\begin{aligned}
R_{\theta}(q) \operatorname{or} I(q) & \cong N\left(p_{\mathrm{av}} V_{\mathrm{S}}\right)^{2}\left(1-\frac{1}{3} q^{2} R_{\mathrm{g}}^{2}\right) \\
& \cong N\left(p_{\mathrm{av}} V_{\mathrm{S}}\right)^{2} \exp \left(-\frac{1}{3} q^{2} R_{\mathrm{g}}^{2}\right)
\end{aligned}
$$

here, $R_{\mathrm{g}}$ is the radius of gyration given by Eq. 17, and the notation of $\left\langle S^{2}\right\rangle^{1 / 2}$ is used in the light scattering field.

$$
R_{\mathrm{g}}^{2}=\int_{0}^{\infty} p(r) r^{2} 4 \pi r^{2} \mathbb{d} r / \int_{0}^{\infty} p(r) 4 \pi r^{2} \mathbb{d} r
$$

Equation 16 indicates that the slope of the $\ln I(q)$ vs. $q^{2}$ plots gives $R_{\mathrm{g}}^{2}$, which is called the Guinier rule or the $q$-rang where such linearity is observed is called the Guinier region. $R_{\mathrm{g}}^{2}$ can be determined for any scattering object as far as $\lim _{q \rightarrow 0} I(q) / c$ is constant. Furthermore, Eq. 16 gives the following for forward scattering:

$$
R_{\theta}(0) \operatorname{or} I(0)=N\left\langle F^{2}(0)\right\rangle_{\mathrm{en}}=N\left\langle V_{\mathrm{S}}^{2} p_{\mathrm{av}}^{2}\right\rangle_{\mathrm{en}}=C \frac{M_{\mathrm{w}}}{N_{\mathrm{A}}}\left(v p_{\mathrm{av}}\right)^{2}
$$

here, $M_{\mathrm{w}}, v$, and $N_{\mathrm{A}}$ are the molar mass of the scattering objects, the specific volume, and Avogadro's number, respectively. The specific volume is determined by density measurements of the solution, the value of $p_{\text {av }}$ can be estimated from the chemical structures in X-ray scattering, and $v p_{\mathrm{av}}$ is the refractive index increment in light scattering. Therefore, $M$ is determined by extrapolating $I(0) / \mathrm{C}$ to the zero concentration. For polydisperse systems, the weightaveraged molecular weight $M_{\mathrm{w}}$ is obtained.

\section{Small-angle X-ray scattering}

\section{Solid sphere model}

Monodisperse solid spheres may not be useful to analyze real systems, but provide a variety of useful insights for the basic understanding of scattering. $F(q)$ in Eq. 15 from a sphere with a radius of $R_{\mathrm{C}}$ and a scattering length of $p_{\mathrm{C}}$ immersed in a solvent with a scattering length of $p_{\text {sol }}$ is 
Fig. 4 Scattering profiles from solid spheres. A The particle scattering function is plotted against the reduced length of $q R_{\mathrm{C}}$, compared with the Guinier expression. B Comparing the profiles with different $R_{\mathrm{C}}$ plotted double-logarithmically. The slope of -4 is indicated by the straight line to show the Porod rule
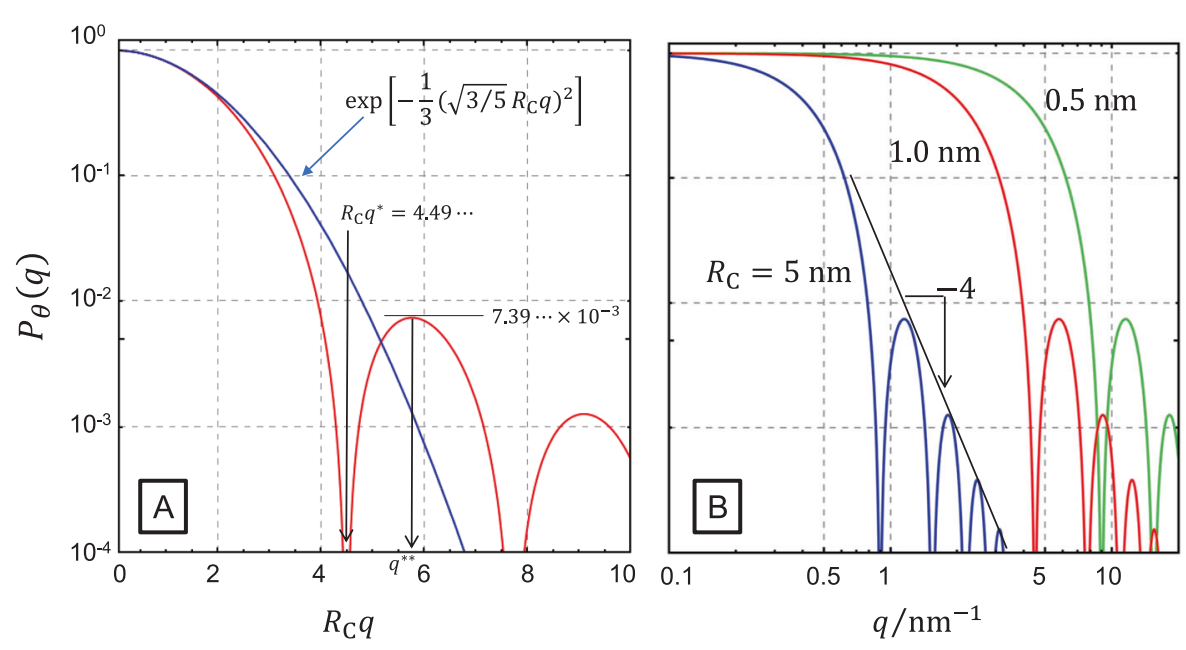

Fig. 5 A representative model of a double-layered core-shell model and its scattering profiles. A Scattering density profile: the solvent scattering length is set to $p_{\text {sol }}=0$. B Comparison of the overall scattering $F^{2}(q)$, where the values are multiplied by $10^{3}$ to facilitate comparison, selfcorrelation terms are $F^{2}$ core and $F_{\text {shell, }}^{2}$, and the cross term is $F_{\text {core }} \times F_{\text {shell }}$. The inset shows negative values in $F_{\text {core }} \times F_{\text {shell }}$

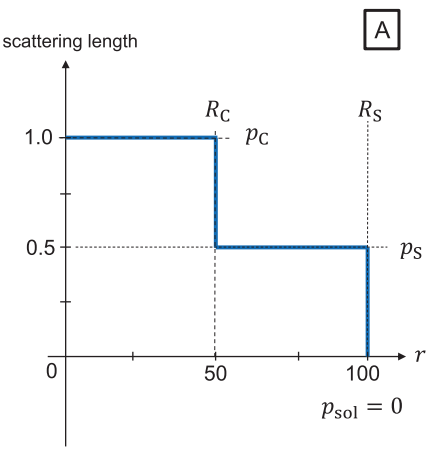

given by:

$$
\begin{aligned}
& F(q)=\left(p_{\mathrm{C}}-p_{\text {sol }}\right) V_{\mathrm{C}} \phi\left(q R_{\mathrm{C}}\right) \\
& \phi(x)=3 \frac{(\sin x-x \cos x)}{x^{3}}
\end{aligned}
$$

where $V_{\mathrm{C}}$ is the volume of the sphere. The forward scattering is $F^{2}(0)=\left(p_{\mathrm{C}}-p_{\text {sol }}\right)^{2} V_{\mathrm{C}}^{2}$ because of $\phi(0)=1$. The red line in Fig. 4A plots the particle scattering function $P_{\theta}(q)$ against $q R_{\mathrm{C}}$, compared with the Guinier rule. The Guinier expression of Eq. 16 is a good approximation at $q R_{\mathrm{C}}<1-2$, and such a region is called the Guinier region. For solid spheres, $R_{\mathrm{g}}=$ $\sqrt{3 / 5} R_{\mathrm{C}}$ is held. The first intensity minimum position is given by $R_{\mathrm{C}} q^{*}=4.49 \cdots$. When the first peak top value is expressed by $F^{2}\left(q^{* * *}\right)$, any solid sphere gives the relation $F^{2}\left(q^{* * *}\right) / F^{2}(0)=$ $7.39 \cdots \times 10^{-3}$. Therefore, if a scattering profile from an experiment shows the first peak and the Guinier region and satisfies this relation, a solid sphere might be a good model for the scattering object. Figure 4B shows a double-logarithmic plot for $P_{\theta}(q)$ and $q$ for three different sizes. With an increase in $R_{\mathrm{C}}$, the entire profile is shifted parallel to the smaller $q$. The peak top intensity decays in the manner of $I \propto q^{-4}$, reflecting the discrete change in the scattering length at the interface between the scattering object and the solvent (i.e., sharp interface). Incidentally, the scattering intensity follows the relation $I(q) \propto q^{-\beta}$ at large $q$, which is called the Porod rule. $\beta=4$ for the sharp interface, $4>\beta>3$ for the fractal surface, and $\beta>4$ for the diffused interface.

\section{Double-layered models}

The simplest model for spherical core-shell micelles may be a two-layered model that has two constant scattering length densities, $p_{S}$ and $p_{C}$, and the corresponding two radii, $R_{\mathrm{C}}$ and $R_{S}$, as shown in Figs. 1 and 5A. Although this model is very simple, the scattering from most polymeric and lipid micelles can be fitted quite well by this model. The structural amplitude $F(q)$ can be given by Eq. 20:

$$
F(q)=\left(p_{\mathrm{C}}-p_{\mathrm{S}}\right) V_{\mathrm{C}} \phi\left(q R_{\mathrm{C}}\right)+\left(p_{\mathrm{S}}-p_{\text {sol }}\right) V_{\mathrm{S}} \phi\left(q R_{\mathrm{S}}\right) \equiv F_{\text {core }}+F_{\text {shell }}
$$



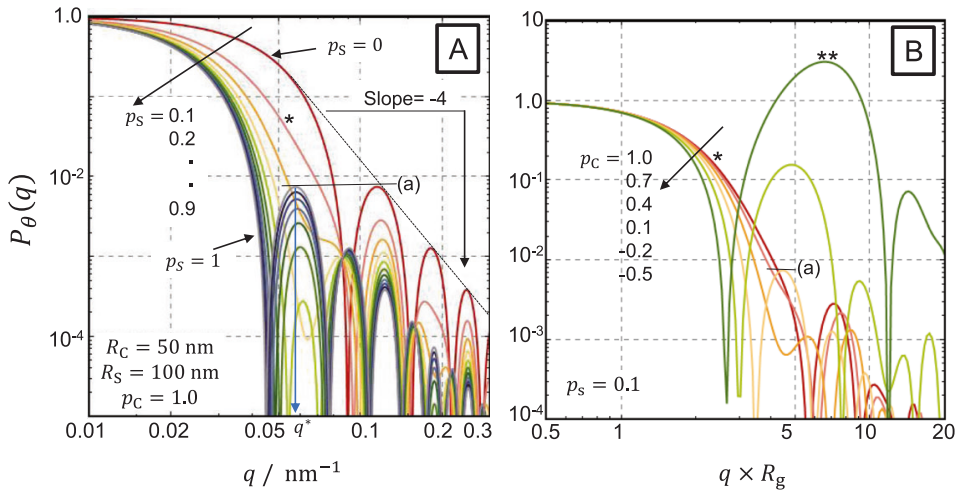

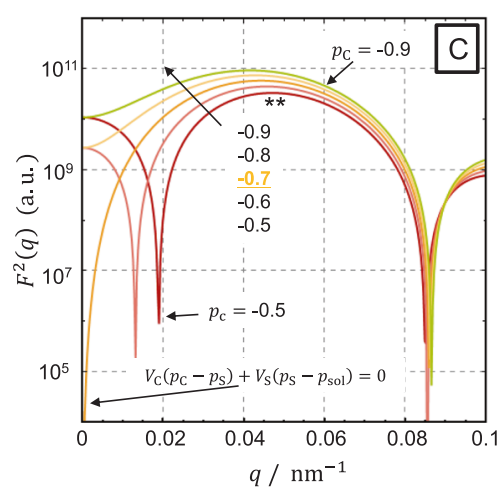

negative and $p_{S}=0.1 ; p_{C}$ is changed from 0.1 to -0.5 . Asterisk shows the same profiles in A, B. C Profiles change in the vicinity of where $\lim _{q \rightarrow 0} F^{2}(q)=0, p_{C}=-0.7$, under the conditions of $p_{S}=0.1$ here, $V_{\mathrm{C}}$ and $V_{\mathrm{S}}$ are the core and micelle volumes and, comparing Eqs. 19 and 20, $F(q)$ of a double-layered sphere is seen to be given by the summation of two solid spheres after appropriate weighting. The forward scattering is $F^{2}(0)=\left[p^{\prime}{ }_{\mathrm{C}} V_{\mathrm{C}}+\left(V_{\mathrm{S}}-V_{\mathrm{C}}\right) p_{\mathrm{S}}^{\prime}\right]^{2}$ by using the excess scattering $p^{\prime}$. After taking the square of $F(q)$, there are three terms: $F_{\text {core }}^{2}, F_{\text {shell }}^{2}$, and $F_{\text {core }} \times F_{\text {shell }}$. The first two terms relate to the self-correlation of each domain and the last term relates to the cross-correlation between the core and shell domains. Figure 5B plots $F^{2}(q)$ against $q$ for the case of $R_{\mathrm{C}}=50 \mathrm{~nm}, R_{\mathrm{S}}=100 \mathrm{~nm}, p_{S}=1.0$, and $p_{C}=0.5$, comparing the self- and cross-correlations. As shown in the inset, the cross term provides negative values at a certain $q$ range. The overall scattering intensity $F^{2}(q)$ is given as a result of summation of these three terms. Figure 6 demonstrates how $P_{\theta}(q)$ changes as $p_{\mathrm{S}}$ and $p_{\mathrm{C}}$ are changed. Panel A shows changes when $p_{\mathrm{S}}$ was changed from 0 to 1 , with the constant $p_{\mathrm{C}}=1.0$. The double-layered spheres with positive $p_{\mathrm{S}}$ and $p_{\mathrm{C}}$ always give $F^{2}\left(q^{*}\right) / F^{2}(0)<7.39 \cdots \times 10^{-3}$, as indicated in Panel A. As shown in Panel B, when $p_{\mathrm{C}}$ becomes negative, $F^{2}\left(q^{*}\right) / F^{2}(0)>7.39 \cdots \times 10^{-3}$, and, for some cases, $F^{2}\left(q^{*}\right)>F^{2}(0)$. Therefore, comparing $F^{2}\left(q^{*}\right)$ and $F^{2}(0)$, we can roughly estimate the magnitude of the electron density in each layer. When the condition $V_{\mathrm{C}}\left(p_{\mathrm{C}}-\right.$ $\left.p_{\mathrm{S}}\right)+V_{\mathrm{S}}\left(p_{\mathrm{S}}-p_{\text {sol }}\right)=0$ is fulfilled, that is, $p_{\mathrm{av}}=0$ or is close to this condition, $F^{2}(q)$ becomes zero at the low angle, as shown in $\mathrm{C}$ (at $p_{\mathrm{C}}=-0.7$ ). Therefore, no Guinier region is observed. This situation can occur in the case where the core consists of alkyl chains whose electron density is lower than that of aqueous solutions [50]. Incidentally, as far as the Guinier region is observed, $R_{\mathrm{g}}$ for the double-layered model is given by:

$R_{\mathrm{g}}^{2}=\frac{3\left[V_{\mathrm{C}} R_{\mathrm{C}}^{2}\left(p_{\mathrm{C}}-p_{\mathrm{S}}\right)+V_{\mathrm{S}} R_{\mathrm{S}}^{2}\left(p_{\mathrm{S}}-p_{\mathrm{sol}}\right)\right]}{5\left[V_{\mathrm{C}}\left(p_{\mathrm{C}}-p_{\mathrm{S}}\right)+V_{\mathrm{S}}\left(p_{\mathrm{S}}-p_{\mathrm{sol}}\right)\right]}$
In the simple core-shell model, the local chain-segment density is assumed to be constant in both core and shell regions. Although a large amount of experimental data can be fitted by this model, we may sometimes need a slightly more elaborate model. As shown in Fig. 2, the crowding of the shell chains may be classified into two types. In the case of the "overcrowding condition", the local chainsegment density is a function of the radial distance $r$ and thus $p_{\mathrm{S}}$ as well. In such a case, $p_{\mathrm{S}}(r)$ is considered a decreasing function of $r$ because the chain crowding decreases towards the surface. In the scaling model, this feature is expressed by increasing the blob size, as shown in Figs. $2 \mathrm{c}$ and 7A. The general expression is given as follows:

$$
p_{\mathrm{S}}(r)=\left(p_{\mathrm{S} . \text { inner }}-p_{0}\right)\left(\frac{r}{R_{\mathrm{C}}}\right)^{b}+p_{0}
$$

The value of $b$ changes from 0 to $2 . b=0$ represents the simple core-shell model and $-4 / 3$ corresponds to the case of a good solvent for the swollen state derived by Daoud and Cotton [25, 51]. Figure 7A illustrates the scattering density profile and Fig. 7B compares scattering profiles between $b=0$ and $-4 / 3$. In this calculation, we used $p_{\text {S.inner }}=0.5$ (upper) and $=0.2$ (lower) and, for each case, the value of $p_{S}$ for $b=0$ was chosen to satisfy the condition that the integrated scattering lengths over the shell are identical.

When $p_{S}-p_{0}$ is large enough compared with $p_{C}-p_{0}$ (upper), a significant difference between the constant and decaying shells appears in the range of $q=0.05-0.1 \mathrm{~nm}^{-1}$, while there is no notable difference in the Guinier region $\left(q<0.05 \mathrm{~nm}^{-1}\right)$. When $p_{S}-p_{0}$ is small, there is not so much difference in the entire profile. Irrespective of how we choose the value of $b$, we always have the slope of -4 in the Porod region. 
Fig. 7 Schematic illustration of the polymeric micelles at the overcrowded state in shell chains, built on the analogy with the star polymers and a scattering density profile following Daoud-Cotton's swollen state (A). Comparison of the scattering profiles between the constant (green) and decaying (red) shells for $p_{\text {S.inner }}=0.5$ (upper) and $=0.2$ (lower) (B)
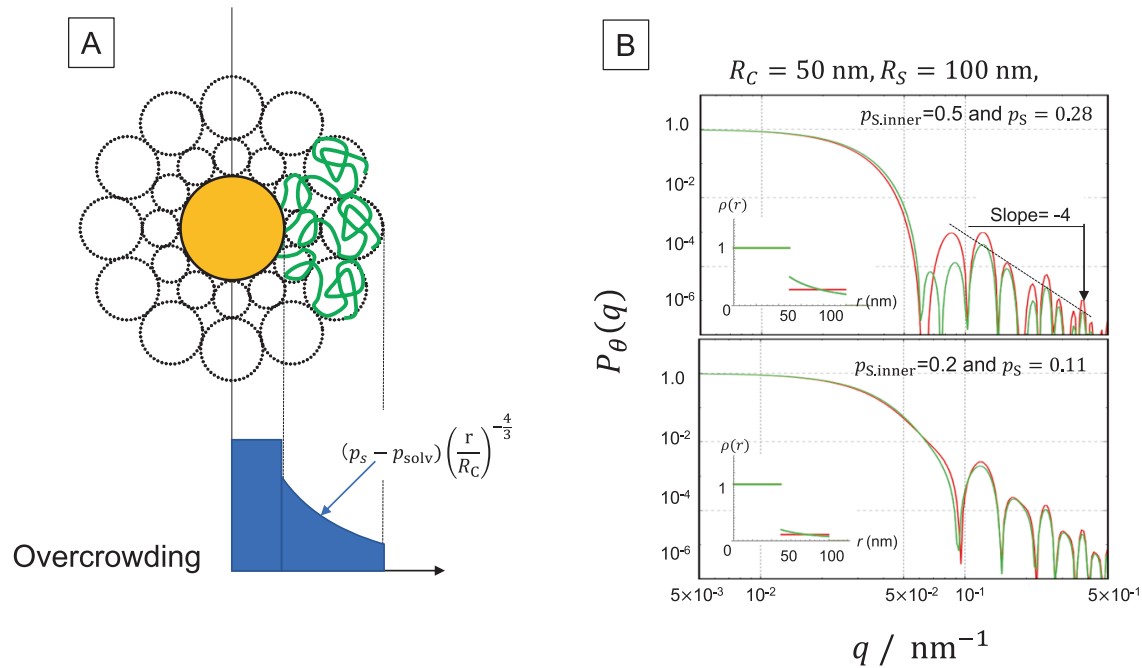

\section{Pedersen's model for polymeric micelles}

Some polymeric micelles show a slope of -2 instead of -4 in the Porod region. These are normally cases for small $N_{\mathrm{agg}}$ and long $L_{\mathrm{S}}$ relative to $L_{\mathrm{C}}$ and this characteristic Porod exponent can be ascribed to scattering from flexible shell chains. Debye calculated $P_{\theta}(q)$ for a random coil with the number of segments $N$ and the Kuhn segment length $a$ as follows:

$$
\begin{aligned}
P_{\theta . \text { Debye }}(x) & =\frac{2}{x^{2}}[\exp (-x)-1+x] \\
x & =\frac{1}{6} N a^{2} q^{2}=R_{\mathrm{g}, \text { coil }}^{2} q^{2}
\end{aligned}
$$

here, $R_{\mathrm{g} . \text { coil }}^{2}$ is the radius of gyration of the Gaussian chain. Figure 8 presents the $q$-dependence of Eq. 23, showing that the intensity decays in the manner of -2 in the Porod region. This slope is characteristic of the random coil chains in which the distance distribution function is followed by the Gaussian random walk statistics.

Pedersen and Gerstenberg proposed a model for blockcopolymer micelles by taking account of the scattering from shell chains [52-54]. This model, hereinafter referred to as the PG model, consists of a spherical core having uniform density and Gaussian shell chains attached to the core surface. The PG model is based on the following two assumptions: (i) The center of mass of each shell chain is separated by the radius of gyration of the shell chain $\left(R_{\mathrm{g} . \mathrm{sh}}\right)$ from the core surface (Fig. 9B). This was supported by Monte Carlo simulation, although there was still overlap between the shell chains and core. In addition, (ii) the shell chains are continuously (evenly) distributed on the spherical core surface. However, the actual electron density in the corona chain region is not always laterally homogeneous due to self-avoidance and mutual interaction.

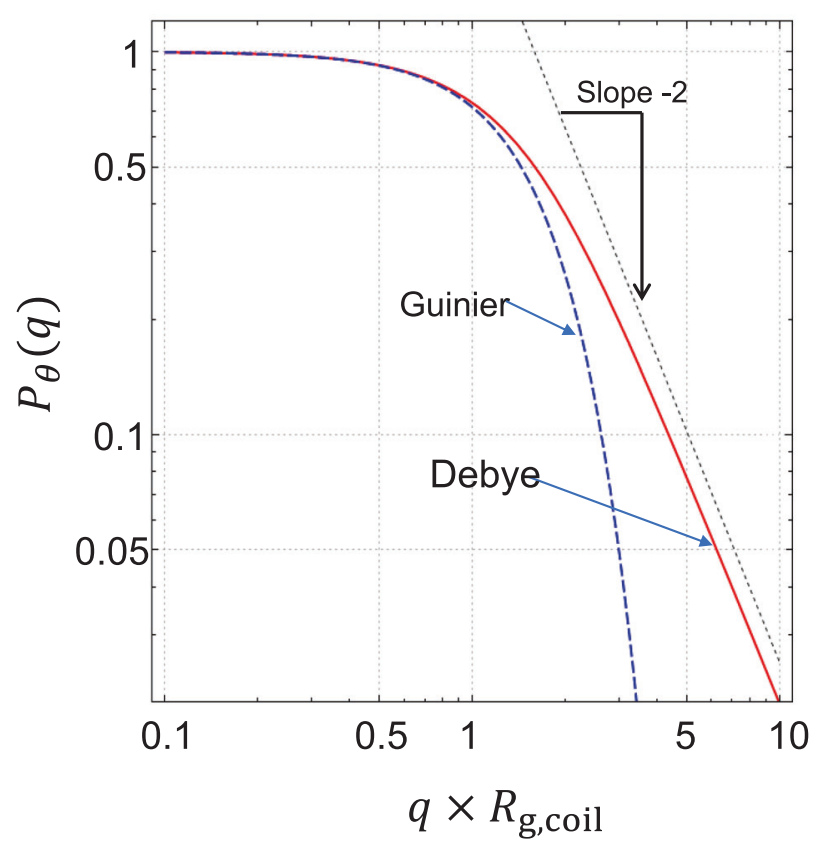

Fig. 8 Scattering profile from the Gaussian chain, compared with the Guinier expression

The particle scattering function $P_{\theta \text {,mic }}(q)$ from a polymeric micelle with aggregation number $N_{\text {agg }}$, radius of the core $R_{\mathrm{C}}$ with the excess scattering length $\rho_{\mathrm{C}}$, and excess scattering length $\left(\rho_{\mathrm{S}}\right)$ of the shell chains with radius of gyration $R_{\text {g.sh }}$ is given by:

$$
\begin{aligned}
P_{\theta, \text { mic }}(q)= & \frac{1}{\left(\rho_{\mathrm{C}}+\rho_{\mathrm{S}}\right)^{2}}\left[\rho_{\mathrm{C}}^{2} f_{\mathrm{C}}(q)+\rho_{\mathrm{S}}^{2} f_{\mathrm{Sh}}(q)+2 \rho_{\mathrm{C}} \rho_{\mathrm{S}} S_{\mathrm{Co}-\mathrm{Sh}}(q)\right] \\
& F_{\text {mic }}^{2}(q)=N_{\mathrm{agg}}^{2}\left(\rho_{\mathrm{S}}+\rho_{\mathrm{C}}\right)^{2} P_{\theta, \text { mic }}(q)
\end{aligned}
$$

here, $f_{\mathrm{C}}(q)$ is the self-correlation of the core given by $f_{\mathrm{C}}(q)=\Phi(q)^{2}$ using Eq. 19. There is a dimensional 
Fig. 9 A schematic illustration calculating the interference terms for two infinitely thin shells separated by the distance of $r(\mathbf{A})$ and the polymeric micellar model having several random coils as shell chains and its electron density profiles (B)
A

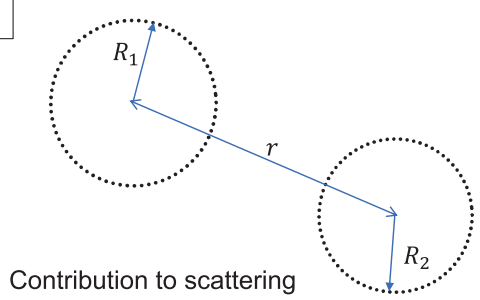

$\frac{\sin q R_{1}}{q R_{1}} \times \frac{\sin q R_{2}}{q R_{2}} \times \frac{\sin q r}{q r}$
B

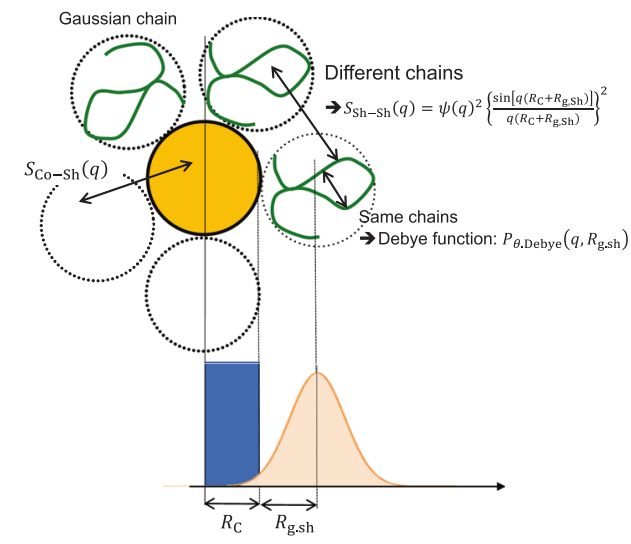

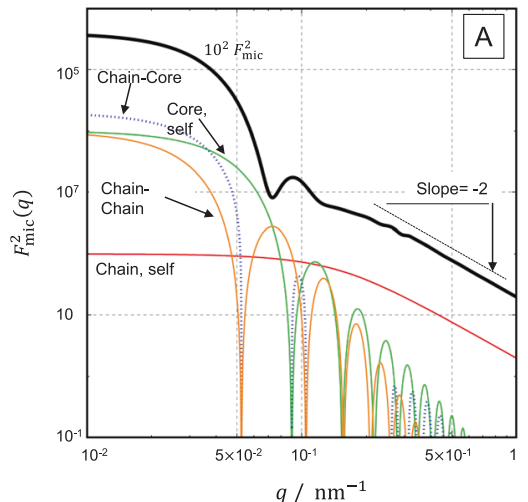

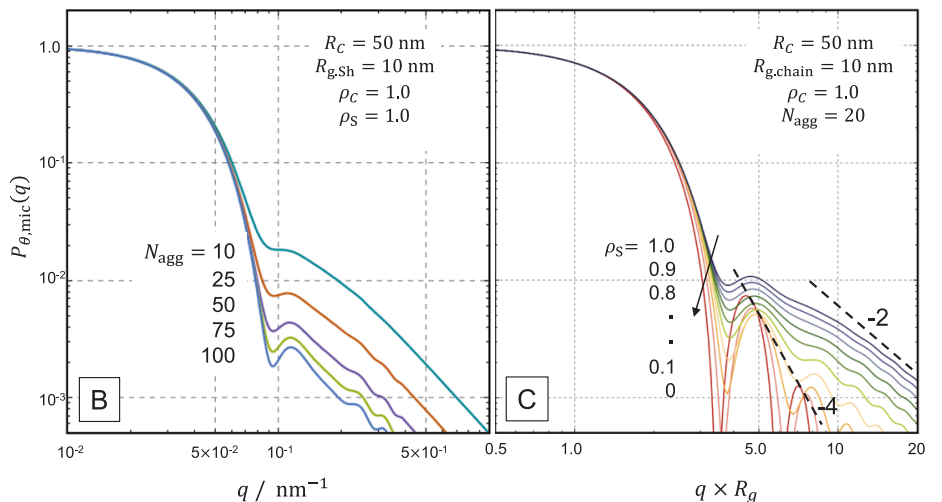

Fig. 10 Scattering profiles from a typical example of the PG model $R_{\mathrm{C}}=40 \mathrm{~nm}, R_{\mathrm{g}, \mathrm{Sh}}=10 \mathrm{~nm}, N_{\mathrm{agg}}=100, \rho_{\mathrm{C}}=\rho_{\mathrm{S}}=1$ (A). How $N_{\mathrm{agg}}$ and $\rho_{\mathrm{S}}$ alter the profiles as $N_{\mathrm{agg}}$ changes from 10 to $100(\mathbf{B})$ and as $\rho_{\mathrm{S}}$ changes from 1.0 to $0(\mathbf{C})$

difference in the excess scattering length between the PG model and the core-shell model. $f_{\mathrm{Sh}}(q)$ is defined as:

$f_{\mathrm{Sh}}(q) \equiv \frac{P_{\theta \text {.Debye }}\left(q, R_{\mathrm{g} . \mathrm{sh}}\right)}{N_{\mathrm{agg}}}+\frac{\left(N_{\mathrm{agg}}-1\right) S_{\mathrm{Sh}-\mathrm{Sh}}(q)}{N_{\mathrm{agg}}}$

$S_{\mathrm{Sh}-\mathrm{Sh}}(q)$ in Eq. 25 is the interference factor between different shell chains given by:

$S_{\mathrm{Sh}-\mathrm{Sh}}(q)=\psi(q)^{2}\left\{\frac{\sin \left[q\left(R_{\mathrm{C}}+R_{\mathrm{g}, \mathrm{Sh}}\right)\right]}{q\left(R_{\mathrm{C}}+R_{\mathrm{g}, \mathrm{Sh}}\right)}\right\}^{2}$

here, $\psi(q)$ is the scattering amplitude of the shell chain expressed by:

$\psi(q)=\frac{1-\exp \left(-q^{2} R_{\mathrm{g}, \mathrm{Sh}}^{2}\right)}{q^{2} R_{\mathrm{g}, \mathrm{Sh}}^{2}}$ where $\psi(q)$ can be derived from:

$\psi(q)=\frac{1}{L} \int_{0}^{L} \exp \left[-\frac{a^{2}}{6} l q^{2}\right] \mathbb{d} l$

This means that the integration is carried out over the points along the contour length of $L=N a$ of the chain. The $S_{\mathrm{Co}-\mathrm{Sh}}(q)$ term in Eq. 24 is given by

$S_{\mathrm{Co}-\mathrm{Sh}}(q)=\phi(x) \psi(q) \frac{\sin \left[q\left(R_{\mathrm{C}}+R_{\mathrm{g}, \mathrm{Sh}}\right)\right]}{q\left(R_{\mathrm{C}}+R_{\mathrm{g}, \mathrm{Sh}}\right)}$

To obtain Eqs. 26 and 29, it is convenient to consider two infinitely thin shells of radii $R_{1}$ and $R_{2}$ separated by the distance $r$ as shown in Fig. 9A. The interference term for the scattering from these two shells is given by the threefold multiplication of the Debye function.

$\frac{\sin q R_{1}}{q R_{1}} \times \frac{\sin q R_{2}}{q R_{2}} \times \frac{\sin q r}{q r}$ 
When $R_{1}$ is located on the core and $R_{2}$ is on the shell chain, $r=R_{\mathrm{C}}+R_{\mathrm{g}, \mathrm{Sh}}$ and the first and second terms give $\phi$ $(x)$ and $\psi(q)$, respectively (see Eq. 15). Therefore, we obtain Eq. 29. When both $R_{1}$ and $R_{2}$ are on the different shell chains, the first and second terms give $\psi(q)$ and the calculation of the third term must be averaged over all possible combinations where we choose two different shell chains.

$$
\left\langle\frac{\sin q r}{q r}\right\rangle_{\text {all combination }}=\frac{1}{2 N_{\text {agg }}\left(N_{\text {agg }}-1\right)} \sum_{i=1}^{N_{\text {agg }}} \sum_{j=1(j \neq i)}^{N_{\text {agg }}} \int_{0}^{\pi} \int_{0}^{2 \pi} P_{i j}(\theta, \phi) \frac{\sin \left(q r_{i j}\right)}{q r_{i j}} \mathrm{~d} \Omega
$$

Here, $r_{i j}$ is the distance of the mass center of two chains (see Fig. 11C) and $d \Omega$ is the differential solid angle given by $d \Omega \equiv \sin \theta \mathbb{d} \theta \mathbb{d} \phi$ and $\int_{0}^{\pi} \int_{0}^{2 \pi} P_{i j}(\theta, \phi) \mathbb{d} \Omega=1$. In order to proceed further with the calculation from Eq. 11, we need to know a radial distribution function of the mass center of the $j$-th corona chain: $P_{i j}(\theta, \phi)$, assuming that each corona chain is identical. Thus, $P_{i j}(\theta, \phi)$ does not depend on how the $i$-th chain is chosen. $P_{i j}(\theta, \phi)$ means a probability of finding the mass center of the $j$-th chain at the solid angle of $\Omega$. If we can assume that the chains are randomly distributed on the core surface:

$$
P_{i j}(\theta, \phi)=\frac{1}{4 \pi}
$$

By noticing $r_{j}=2\left(R+R_{\mathrm{g}}\right) \sin (\theta / 2)$, Eq. 32 means that the double integration in Eq. 31 does not depend on how to choose $j$ and $i$. Therefore, the summation in Eq. 31 leads to $2 N_{\text {agg }}\left(N_{\text {agg }}-1\right)$ and then we obtain:

$$
\begin{aligned}
\left\langle\frac{\sin q r}{q r}\right\rangle_{\text {all combination }} & =\frac{1}{4 \pi} 2 \pi \int_{0}^{\pi} \mathbb{d} \theta_{j} \frac{2 \sin \left\{q\left[2\left(R_{\mathrm{C}}+R_{\mathrm{g}, \mathrm{Sh}}\right) \sin \frac{\theta_{j}}{2}\right]\right\}}{q\left[2\left(R_{\mathrm{C}}+R_{\mathrm{g}, \mathrm{Sh}}\right) \sin \frac{\theta_{j}}{2}\right]} \\
\sin \theta_{j} & =\left\{\frac{\sin \left[q\left(R_{\mathrm{C}}+R_{\mathrm{g}, \mathrm{Sh}}\right)\right]}{q\left(R_{\mathrm{C}}+R_{\mathrm{g}, \mathrm{Sh}}\right)}\right\}^{2}
\end{aligned}
$$

This leads to Eq. 26, after multiplying $\psi(q)^{2}$ from the first and second terms of Eq. 30 .

Figure 10A compares $F_{\text {mic }}^{2}(q)$ and its constituent terms. The chain-core cross term related to Eq. 29 gives negative values in a certain $q$ range. The chain-self term (Eq. 23, the Debye function) decays as $I(q) \propto q^{-2}$ in the high $q$ region, while the other terms decay more rapidly as $I(q)$. As a result, the Debye function becomes significant in the Porod region in $F_{\text {mic }}^{2}(q)$. Figures $10 \mathrm{~B}, \mathrm{C}$ show how $N_{\text {agg }}$ and $\rho_{\mathrm{S}}$ alter the profiles, indicating that the smaller $N_{\text {agg }}$ and larger $\rho_{\mathrm{S}}$ tend to emphasize the Gaussian Porod exponent. The PG model assumes that the shell chains are isolated enough to satisfy $\sigma<1$ in Eq. 2. In fact, the parameters chosen in Fig. 10A give $\sigma=1$. To calculate the cases for $\sigma>1$, Svaneborg and
Pedersen incorporated the interference effect between corona chains into the PG mode $[55,56]$. They assumed that shell chains correspond to homopolymer chains in semidilute solution and described the scattering by using the random phase approximation. Hereinafter, this model is called the SP model. We showed that the SP model produces a scattering profile similar to that of of the doublelayered model given by Eqs. 20 and 22, when $N_{\text {agg }}>30, \sigma>$ 1 , and $\rho_{\mathrm{C}}>\rho_{\mathrm{S}}$ [57].

\section{Modified Pedersen's model: discrete Platonic micelle model}

Both the PG and SP models assume the random (or even) distribution of the shell chains on the core surface (Eq. 32). However, this distribution is seemed unlikely when $N_{\text {agg }}$ is small. For example, let us consider the extreme case of $N_{\text {agg }}=2$; two shell chains tend to separate as far as possible due to steric repulsion between them. Therefore, it is most likely that they are located at the two poles of the core. Recently, we added a modification to the PG model by taking into account a preferential configuration of the shell chains [58]. This modification was motivated by our finding of "Platonic micelles". We found that, when $N_{\text {agg }}$ is small enough, the shell chains are arranged on the core surface so as to take the coordinates of the vertices' points of a regular polyhedron or Platonic solid [59-64]. The preferential appearance of these configurations can be interpreted in terms of a geometric problem, which may be related to the mathematical problem of how to cover the surface of a sphere efficiently with multiple spherical caps of equal radius. In geometry, this is called the "best packing on a sphere" or Tammes problem [65, 66]. For example, as illustrated in Fig. $11 \mathrm{~A}$, we can have the maximum coverage $[D(N)]$ with three spherical caps when the center of each cap is located on the vertices of an equilateral triangle touching the sphere. In this case, $D(N)=0.75$. For 12 caps, we can have a larger coverage, namely, $D(N)=0.879$, and the centers of the caps have the same configuration as the vertices of a regular icosahedron. $D(N)$ is not a simple monotonic function of $N$. Figure 11B plots $D(N)$ against the number of caps $N$. For large $N, D(N)$ is almost constant at $0.82-0.83$. On the other hand, for small $N$, larger $D(N)$ values are obtained at certain numbers such as $N=6,12$, and 20. Surprisingly, these numbers coincide with our observed values of $N_{\text {agg }}$ [59-63]. Furthermore, many of numbers agree with the face or vertex numbers of the Platonic solids, which is why we named these micelles "Platonic micelles" [64]. Mathematicians have addressed this Tammes problem, and the coordinate values to give the maximum cover ratio by caps on the spherical surface are available as a function of the number of caps.

Therefore, we may infer that the shell chains are located at the positions corresponding to the solutions of the Tammes problem in spherical micelles, and we calculated a 

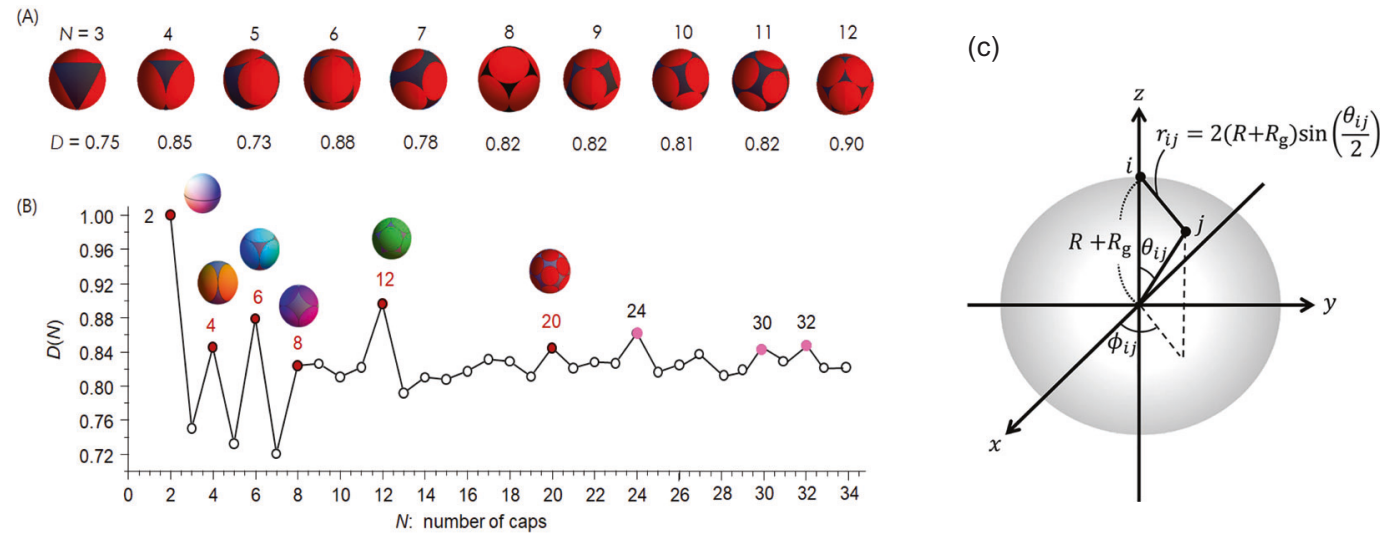

Fig. 11 A The cap configurations when the maximum coverage is attained. $\mathrm{N}$ is the number of identical caps and $\mathrm{D}$ is the maximum coverage. $\mathbf{B}$ The zigzag profile of the maximum coverage against the number of caps. C Illustration of the coordinate values; the points of $i$ and $j$ on a sphere with a radius of $R+R \mathrm{~g}$ indicate the location of the center of the corona chains
Fig. 12 A $S_{\mathrm{Sh}-\mathrm{Sh}}(q) / \psi(q)^{2}$ (Eq. 34) plotted against $q R$ by setting $R_{\mathrm{C}}+R_{\mathrm{g}, \mathrm{Sh}}=R$. a Effect of $N: N=8$ (blue), 40 (red), and 320 (green) without the distortion. $\mathrm{b}$ Effect of the distortion at $N=8: s=0$ (blue), $0.3 \theta \mathrm{min}$ (red), and $0.5 \theta \mathrm{min}$ (green). c Effect of the distortion at $N=40: s=0$ (blue), $0.3 \theta \mathrm{min}$ (red), and $0.5 \theta \min$ (green). B $P_{\theta, \text { mic }}(q)$ for the various $\rho_{\mathrm{C}} / \rho_{\mathrm{S}}$ values of $1,0.2,-0.2,-0.5$, and -1.5 under the constant $R_{\mathrm{C}}+R_{\mathrm{g}}$, $\mathrm{Sh}=30 \mathrm{~nm}$ and constant $\sigma$ value of 0.5 in $N_{\text {agg }}=8$ (a) and 40 (b). The broken black curve represents the PG model, and the broken blue curve stands for the SP model. The solid red, green, and purple curves indicate our proposed discrete model with and without the distortion $\left(s=0,0.3 \theta_{\min }\right.$, and $0.5 \theta_{\min }$, respectively). The curves for $\rho_{\mathrm{C}} /$ $\rho_{\mathrm{S}}=0.2,1,-0.2,-0.5$, and -1.5 are shifted vertically for clarity by multiplying by $10,10^{2}, 10^{3}$ and $10^{4}$ in a, and by $30,10^{3}, 6 \times$ $10^{4}$, and $2 \times 10^{5}$ in $\mathrm{b}$
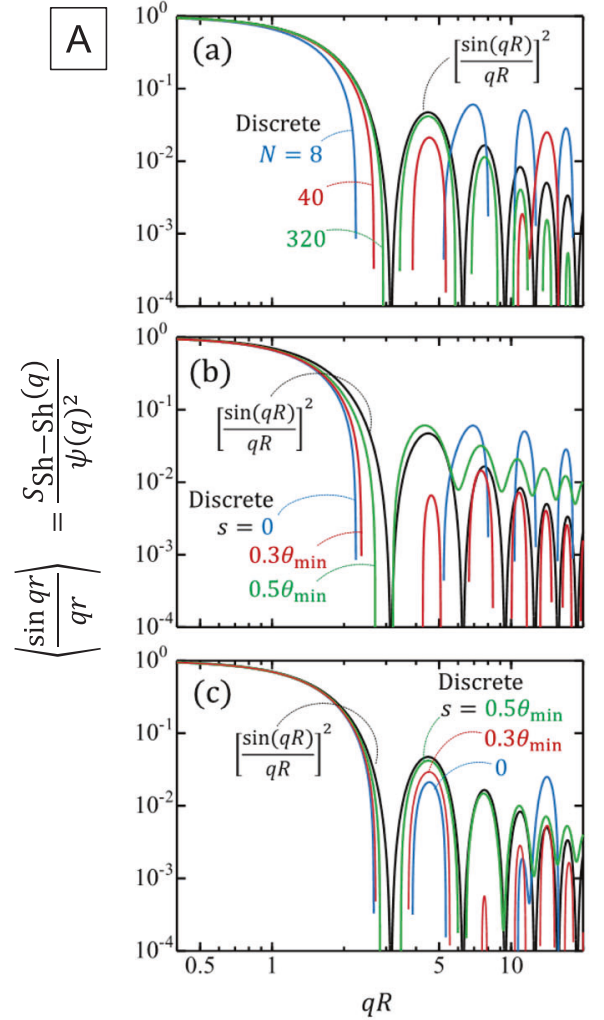

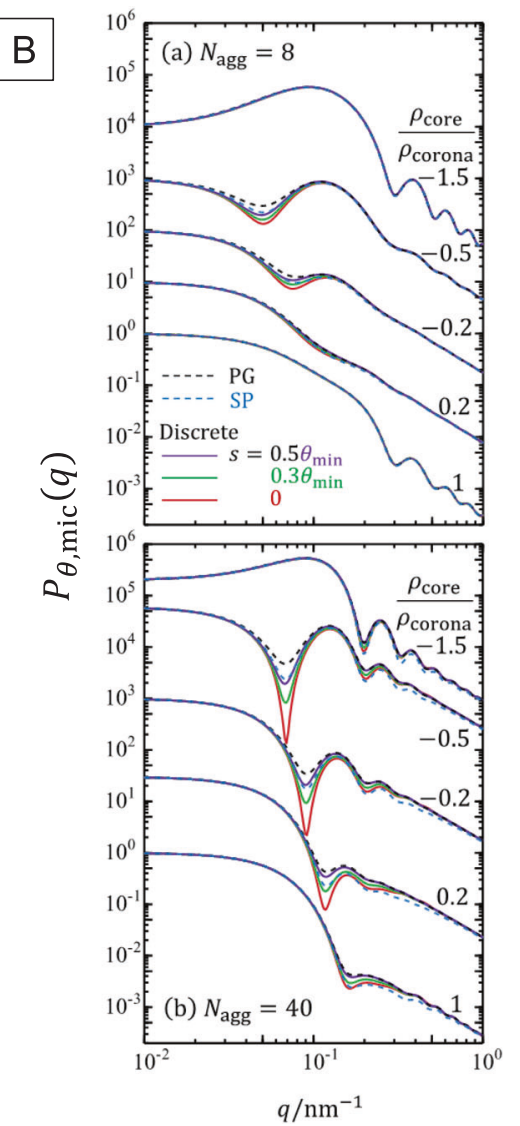

form factor for micelles with the shell chains discretely distributed on the core surface as such. Therefore, $P_{i j}(\theta, \phi)$ is given by as follows:

$P_{i j}(\theta, \phi)=\delta\left(\theta-\theta_{i j}\right) \delta\left(\phi-\phi_{i j}\right)$

here, $\theta_{i j}$ and $\phi_{i j}$ are the angles that satisfy the Tammes problem. $N_{\text {agg }}=1-12$ and 24 , the analytical solutions of the Tammes problem, and thus the coordinate values $\left(\theta_{i j}\right.$, $\left.\phi_{i j}\right)$ in Eq. 31 and $r_{i j}$, are available [67]. However, for other numbers, the analytical solutions and coordinate values have not been reported. Alternatively, the coordinate values can be more easily distributed in a phyllotactic/golden spiral fashion (the points are distributed according to the golden spiral on the spherical surface). In this study, the coordinate values of the phyllotactic/golden spiral fashion were output 

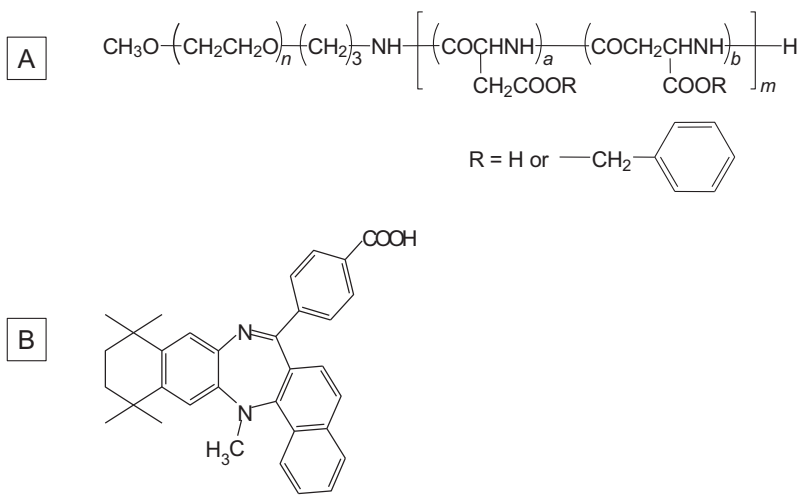

Fig. 13 The chemical structures of poly(ethylene glycol)-block-poly (partially benzyl-esterified aspartic acid) (A) and LE540 (B)

with a command of "SpherePoints" in Mathematica to obtain each $r_{i j}$ value and calculate $S_{\mathrm{Sh}-\mathrm{Sh}}(q)$ by using Eq. 31. For $N_{\text {agg }}=1-12$ and 24 , the solutions of the Tammes problem were used.

The distortion of the location of the corona chains on the core was incorporated by assuming that (i) $\theta_{i j}$, indicated in Fig. 11C, obeys the Gaussian distribution and (ii) the position of each point is independently distorted irrespective of the position of neighboring points. That is, we considered the so-called distortion of the first kind but not that of the second kind in the paracrystal theory. Thus, Eq. 31 for the discrete model can be expressed as

$$
\begin{aligned}
& \left\langle\frac{\sin q r}{q r}\right\rangle_{\text {all combination }}=\frac{1}{N_{\mathrm{agg}}\left(N_{\mathrm{agg}}-1\right)} \sum_{i} \sum_{j(i \neq j)}^{N_{\mathrm{agg}}} N_{\text {agg }} \\
& \left\{\int d \theta \frac{1}{\sqrt{2 \pi s^{2}}} \exp \left[-\frac{\left(\theta-\theta_{i j}\right)^{2}}{2 s^{2}}\right] \frac{\sin \left\{q\left[2\left(R_{\mathrm{C}}+R_{\mathrm{g}, \mathrm{Sh}}\right) \sin \left(\frac{\theta}{2}\right)\right]\right\}}{q\left[2\left(R_{\mathrm{C}}+R_{\mathrm{g}, \mathrm{Sh}}\right) \sin \left(\frac{\theta}{2}\right)\right]}\right\}
\end{aligned}
$$

here, $s$ denotes the standard deviation.

Initially, this discrete model was compared with the PG model in terms of the interference factor between the shell chains location points $\left[S_{\mathrm{Sh}-\mathrm{Sh}}(q) / \psi(q)^{2}\right]$. As shown in Fig. 12A, the curve of the discrete model with a small $N_{\mathrm{agg}}$ shows minima that are too sharp in comparison to $[\sin (q R) /$ $(q R)]^{2}$ (PG model; Eq. 26), although the first minimum position (corresponding to the micellar core radius) of the discrete model is near that of the PG model. As expected, the curve asymptotically becomes close to $[\sin (q R) /(q R)]^{2}$ with increasing $N_{\text {agg }}$, since $[\sin (q R) /(q R)]^{2}$ can be derived with $N_{\text {agg }} \rightarrow \infty$. An increasing $N_{\text {agg }}$ value in this calculation leads to an increase not only in $N_{\text {agg }}$, but also in $\sigma$. Even in $N_{\text {agg }}=8$ and 40 (Fig. 12A, B, C), by increasing $s$ (the distortion degree of $\theta_{i j}$, the discrete model follows the curve of $[\sin (q R) /(q R)]^{2}$ in both $N_{\mathrm{agg}}=8$ and 40 . Here, $\theta_{\text {min }}$ stands for the angle corresponding to the angle between the closest points. This indicates that the density distribution on the spherical core surface becomes similar to the evenly distributed state by distortion.

Figure 12B shows $P_{\theta, \text { mic }}(q)$ calculated in $N_{\text {agg }}=8$ and 40 in the discrete, PG and SP models for various $\rho_{\mathrm{C}} / \rho_{\mathrm{S}}$ values under a constant $\sigma_{\mathrm{C}}$ of 0.5 . When $\rho_{\mathrm{C}} / \rho_{\mathrm{S}}$ is near-zero (or negative but not exceeding -1 ), the deviation is significant. Meanwhile, if the absolute value of $\rho_{\text {core }} / \rho_{\text {corona }}$ is sufficiently larger ( $>1$; i.e., the scattering from the core domain is dominant), less deviation among the models is observed, even with the low $\sigma$.

\section{Some examples from our recent work}

\section{Micelles made from poly(ethylene glycol)-block-poly (partially benzyl-esterified aspartic acid) [68-71]}

\section{Analyzing micellar structures by using scattering}

Poly(ethylene glycol)-block-poly(partially benzyl-esterified aspartic acid), denoted by PEG-P(Asp(Bzl)), is one of the most examined blockcopolymers for drug carriers $[10,72,73]$. Its chemical structure is shown in Fig. 13A. However, little is known about the fundamental physical properties of PEG-P(Asp(Bzl)). Eighteen samples of PEG-P (Asp(Bzl)) with different benzylation fractions (Bzl\%), aspartic chain lengths ( $\mathrm{m}$ or $\left.L_{\mathrm{C}}\right)$ and PEG chain lengths $\left(M_{\mathrm{w}}\right.$, ${ }_{\mathrm{PEG}}=5200$ and 12,000) were synthesized. We prepared micelles from them, and the sample codes are listed in Table 1 . Here, $\mathrm{H}$ in the sample code stands for the PEG molecular weight; 5 and 12 means $M_{\mathrm{w}, \text { PEG }}=5200$ and 12,000 , respectively. A and B indicate the number of the Asp chain lengths, i.e., $\mathrm{m}$ in Fig. 12A and the benzylation molar \%, respectively. The suffixes $\mathrm{s}$ and $\mathrm{d}$ indicate the difference in how to disperse samples into water: $s$ and $d$ are sonication and dialysis described later. We determined the molecular weight $M_{\mathrm{w}}$ and the radius of gyration $\left\langle S^{2}\right\rangle_{z}^{1 / 2}$ by using light scattering coupled with FFF. An example of the applied cross-flow profile is presented in Fig. 14A, and the elution fractgrams recorded with $\mathrm{LS}$ at $90^{\circ}$ and $\mathrm{RI}$ are shown in Fig. 14B for H5-A27-B83-s. As shown in A, the cross-flow rate exponentially decreased and finally linearly decreased to 0. FFF makes smaller particles (i.e., smaller hydrodynamic volumes) transport faster and thus elute prior to larger particles. The peak at $8 \mathrm{~min}$ can be assigned to the polymeric micelles. From this peak, we obtained $M_{\mathrm{w}}$ and $\left\langle S^{2}\right\rangle_{z}^{1 / 2}$ as well as $N_{\text {agg }}$ as summarized in Table 1.

Figure 15A shows a typical SAXS profile from PEG-P (Asp(Bzl)) samples, after the intensities were extrapolated to zero concentration. We used a spherical core-shell model given by Eq. 20 to fit the data. We did not need more elaborate models in our analysis because we did not observe the slope of -2 due to the Gaussian behavior of the shell 
Table 1 Micellar characters of PEG-P(Asp(Bzl)) samples determined by SLS-coupled with FFF and SAXS
Fig. 14 Field-flow fractionation (FFF) and light scattering (LS) measurements. A An example of the cross-flow profile. B The resultant fractgrams detected with LS at $90^{\circ}$ and RI (reflective index). The RI intensities were converted to concentrations. $\mathbf{C}$ The Berry plots for all samples of $\mathrm{H} 5$ series at the top of the RI peaks in $\mathbf{B}$

\begin{tabular}{lccccccc}
\hline Micelle sample code & $10^{-5} M_{w}{ }^{\mathrm{LS}}$ & $N_{\mathrm{agg}}{ }^{\mathrm{LS}, \mathrm{NMR}}$ & $\frac{\left\langle S^{2}\right\rangle_{2}^{1 / 2 \mathrm{LS}}}{\mathrm{nm}}$ & $\frac{R_{\mathrm{g}}^{\mathrm{SAXS}}}{\mathrm{nm}}$ & $\frac{R_{\mathrm{C}}}{\mathrm{nm}}$ & $\frac{R_{\mathrm{S}}}{\mathrm{nm}}$ & \multicolumn{1}{c}{$\sigma$} \\
\hline H5-A20-B84-s & 3.25 & 35.8 & $15 \pm 5$ & 7.4 & 4.3 & 10.2 & 4.3 \\
H5-A24-B84-s & 4.78 & 49.0 & $9 \pm 3$ & 7.4 & 4.8 & 11.5 & 4.8 \\
H5-A24-B83-s & - & - & - & 6.5 & 5.9 & 12.5 & - \\
H5-A26-B77-s & 3.82 & 37.8 & $11 \pm 4$ & 7.6 & 4.2 & 10.2 & 4.2 \\
H5-A26-B81-s & 4.24 & 41.5 & $11 \pm 4$ & 6.7 & 4.4 & 10.2 & 4.4 \\
H5-A26-B89-s & 10.4 & 100.2 & $12 \pm 4$ & 9.4 & 5.7 & 12.0 & 5.7 \\
H5-A27-B66-s & 2.77 & 27.9 & $9 \pm 3$ & 8.1 & 3.9 & 9.3 & 3.9 \\
H5-A27-B83-s & 7.69 & 74.3 & $12 \pm 4$ & 7.1 & 5.4 & 10.2 & 5.4 \\
H5-A27-B83-d & 7.02 & 67.9 & $12 \pm 3$ & 7.9 & 5.4 & 11.8 & 5.4 \\
H5-A30-B82-s & 3.55 & 32.7 & $11 \pm 3$ & 6.5 & 4.7 & 11.1 & 4.7 \\
H5-A32-B77-s & 3.58 & 32.1 & $12 \pm 4$ & 6.1 & 4.3 & 10.2 & 4.3 \\
H12-A10-B84-s & 6.9 & 50 & $8 \pm 3$ & 8.2 & 3.2 & 12.5 & 4.2 \\
H12-A17-B83-s & 13.6 & 90 & $7 \pm 3$ & 7.3 & 3.8 & 13.0 & 6.7 \\
H12-A21-B85-s & 15.9 & 100 & $8 \pm 3$ & 7.8 & 4.0 & 13.0 & 7.5 \\
H12-A30-B88-s & 73.9 & 282 & $12 \pm 1$ & 11.6 & 6.4 & 22.0 & 11.1 \\
H12-A36-B88-s & 50.1 & 390 & 17 & 16.9 & 7.4 & 18.0 & 4.4 \\
H12-A42-B89-s & 139 & 687 & 22 & 22.0 & 9.2 & 22.0 & 10.7 \\
H12-A49-B89-s & 253 & $1.17 \times 10^{4}$ & 28 & 28.5 & 9.7 & 28.0 & 8.8 \\
\hline
\end{tabular}

LS: determined by MALS coupled with FFF

NMR: used the molecular weight of block-copolymer determined by ${ }^{1} \mathrm{H}-\mathrm{NMR}$

SAXS: determined by SAXS
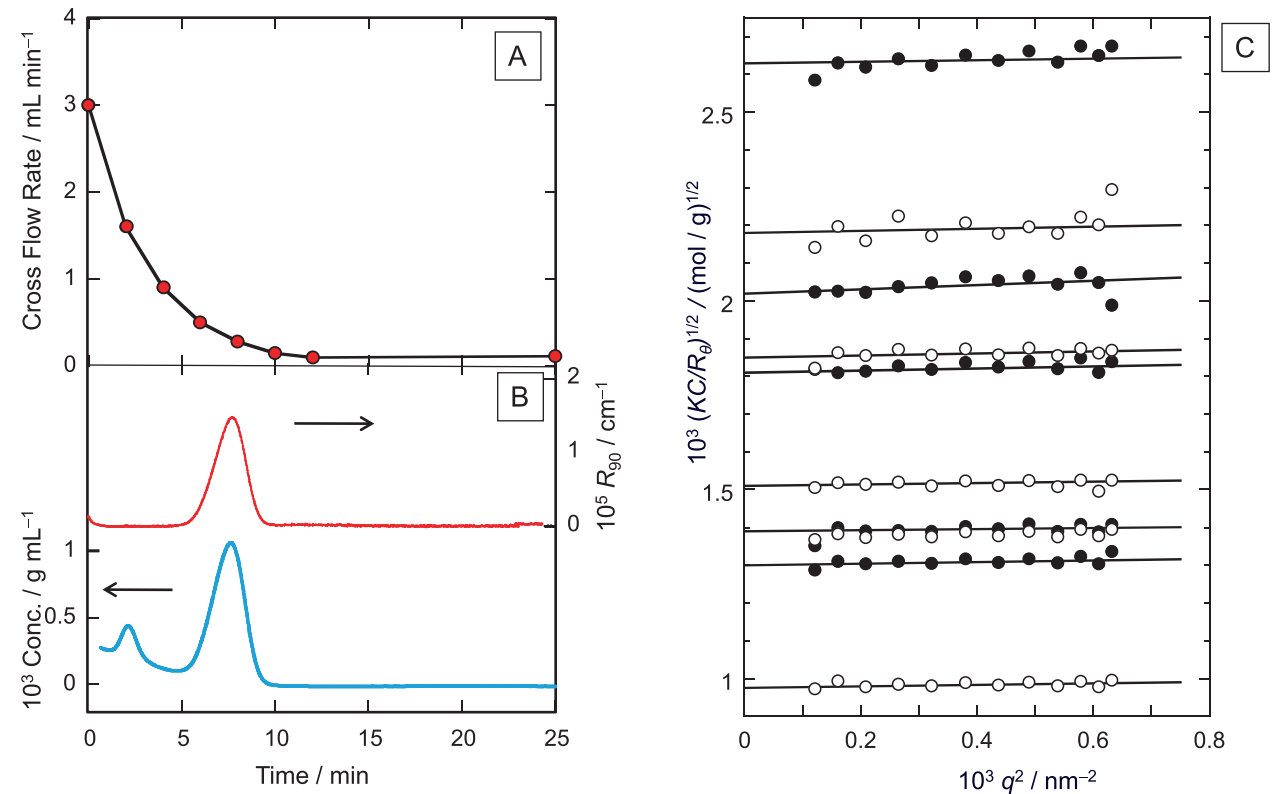

PEG chains and we found that diffused diffraction caused ordering of the shell chains (see later). The core is composed of only $\mathrm{P}(\mathrm{Asp}(\mathrm{Bzl}))$ and thus the scattering length $\rho_{C}$ can be evaluated to be $489 \mathrm{e} / \mathrm{nm}^{3}$ by combining the bulk density of $\mathrm{P}(\mathrm{Asp}(\mathrm{Bzl}))\left(\mathrm{ca} .1 .30 \mathrm{~g} \mathrm{~cm}^{-3}\right)$ with its chemical structure. Because we used water as the solvent, we chose $\rho_{\text {sol }}=334 \mathrm{e} / \mathrm{nm}^{3}$. The electron density of PEG in the amorphous state is $369 \mathrm{e} / \mathrm{nm}^{3}$. Because the PEG blocks are dissolved in water, $\rho_{\mathrm{S}}$ must take a value between 334 and $369 \mathrm{e} / \mathrm{nm}^{3}$. These are the constraining conditions for the scattering lengths. Furthermore, the two geometrical parameters $R_{\mathrm{C}}$ and $R_{\mathrm{S}}$ must satisfy Eq. 21 with $R_{\mathrm{g}}=6.4 \mathrm{~nm}$, which had been determined by the Guinier plot shown in B. Under these conditions, we can perform the fitting analysis 

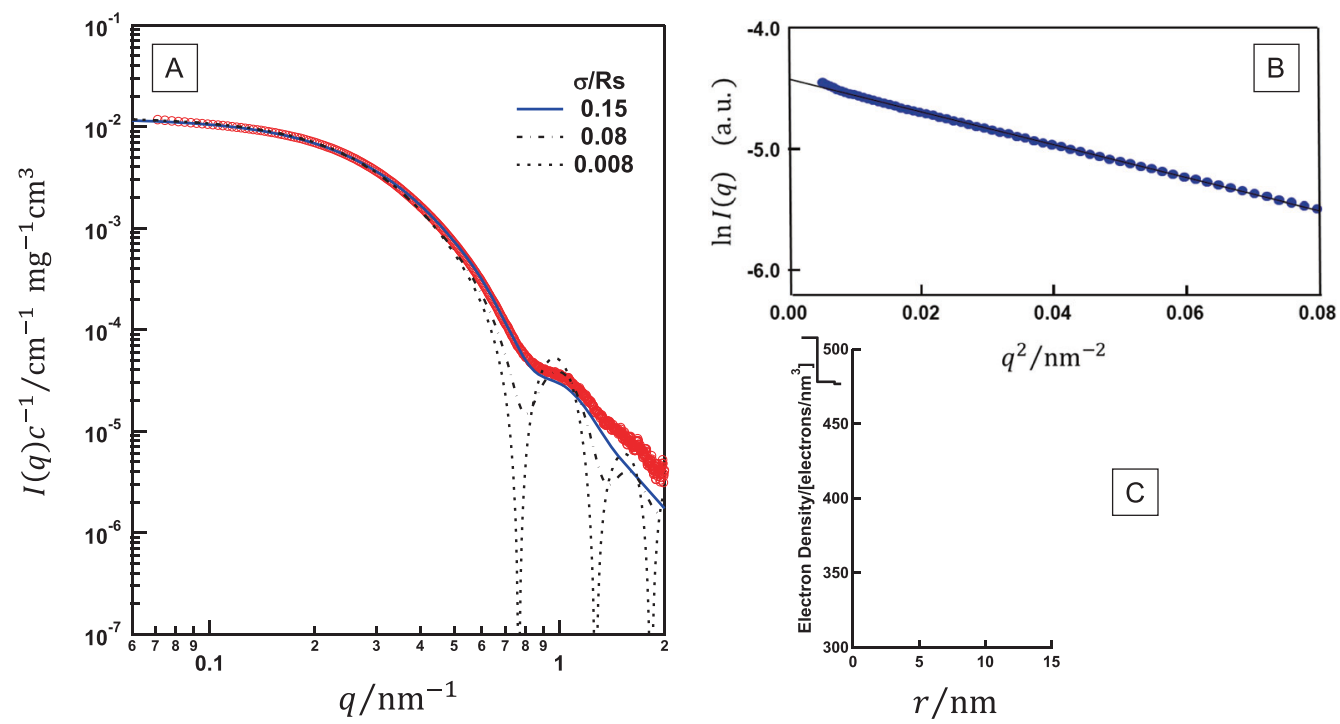

Fig. 15 Comparison of experimental SAXS profile from H5-A24-B83-s and theoretical curves calculated from core-shell sphere model taking account of polydispersity

Fig. 16 Comparison of FFF fractgrams and SAXS profiles between the micelles made the same sample but with different procedure
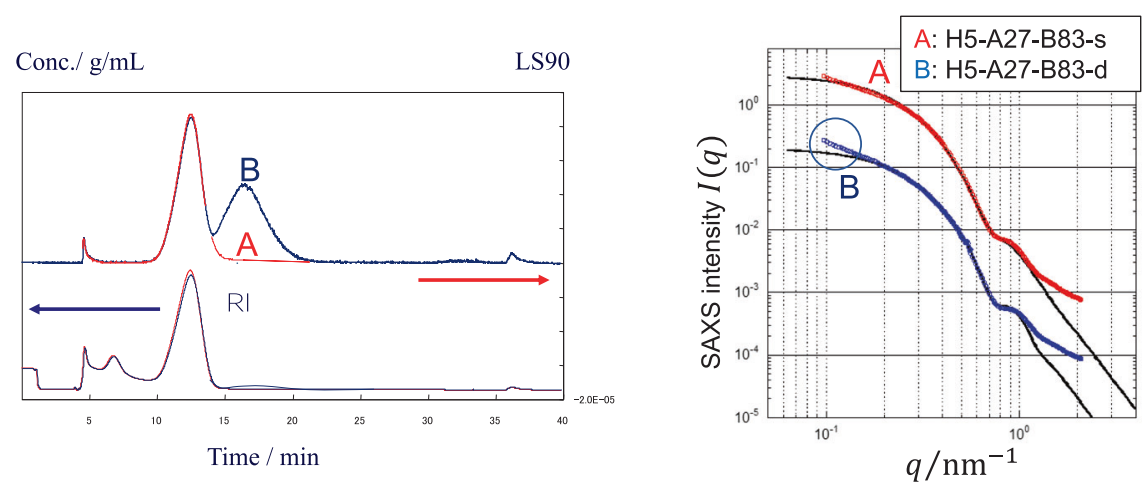

to the SAXS profile by the use of only three adjustable parameters of $R_{\mathrm{C}}, R_{\mathrm{S}}$, and $\rho_{\mathrm{S}}$, which lead to $R_{\mathrm{C}}=5.9 \pm$ $0.1 \mathrm{~nm}$ and $R_{\mathrm{S}}=12.5 \pm 0.3 \mathrm{~nm}$. We assumed that the size distribution can be described by the Gaussian function:

$$
\left\langle F^{2}(q)\right\rangle_{\mathrm{en}}=\frac{1}{\sqrt{2 \pi \sigma}} \int_{R_{C}=-\infty}^{\infty} \exp \left[-\frac{\left(R_{\mathrm{C}}-R_{\mathrm{C}, \mathrm{av}}\right)^{2}}{2 \sigma^{2}}\right] F^{2}\left(q, R_{\mathrm{C}}\right) d R_{\mathrm{C}}
$$

here $F^{2}\left(q, R_{\mathrm{C}}\right)$ is given by Eq. 20 and we took the average over $R_{C}$ and $R_{S}$, independently. Figure 15A shows the final fitting results, and the cross-sectional electron density profile obtained is shown in $\mathrm{C}$. The agreement is quite good except for $q>1.2 \mathrm{~nm}^{-1}$ where the ordering of the $\mathrm{P}$ $(\mathrm{Asp}(\mathrm{Bzl}))$ chains gives an additional diffraction peak (see Fig. 23B). The other samples were analyzed in a similar manner and the results are summarized in Table 1, where in some cases, we treated $\rho_{\mathrm{C}}$ as the fourth adjustable parameter when the original restriction was too strong to obtain a better fitting.
When the micelles were prepared with the same procedure from the same sample, the discrepancy was negligibly small in the FFF chart. However, when we prepared them with a different procedure from the same polymer sample (such as sonication or dialysis), the discrepancy sometimes became large. The normal sonication procedure was as follows: PEG-P(Asp(Bzl)) was dissolved in THF, THF was evaporated and then PEG-P(Asp(Bzl)) was dispersed in an aqueous solvent, and the solution was ultrasonicated. When we used dialysis, THF was exchanged for water using a semipermeable membrane. Figure 16 compares the FFF and SAXS results for these two samples. The FFF from the dialysis sample (B, H5-A27-B83-d) showed a large aggregation peak in the light scattering, but the composition of this peak was less than a few percent judging from RI. On the other hand, SAXS shows a small difference observed only in the low $q$ region. The scattering intensity is proportional to the square of the volume of scattering objects, and thus larger particles may dominate the intensity even though they are minor in composition. In this sense, 


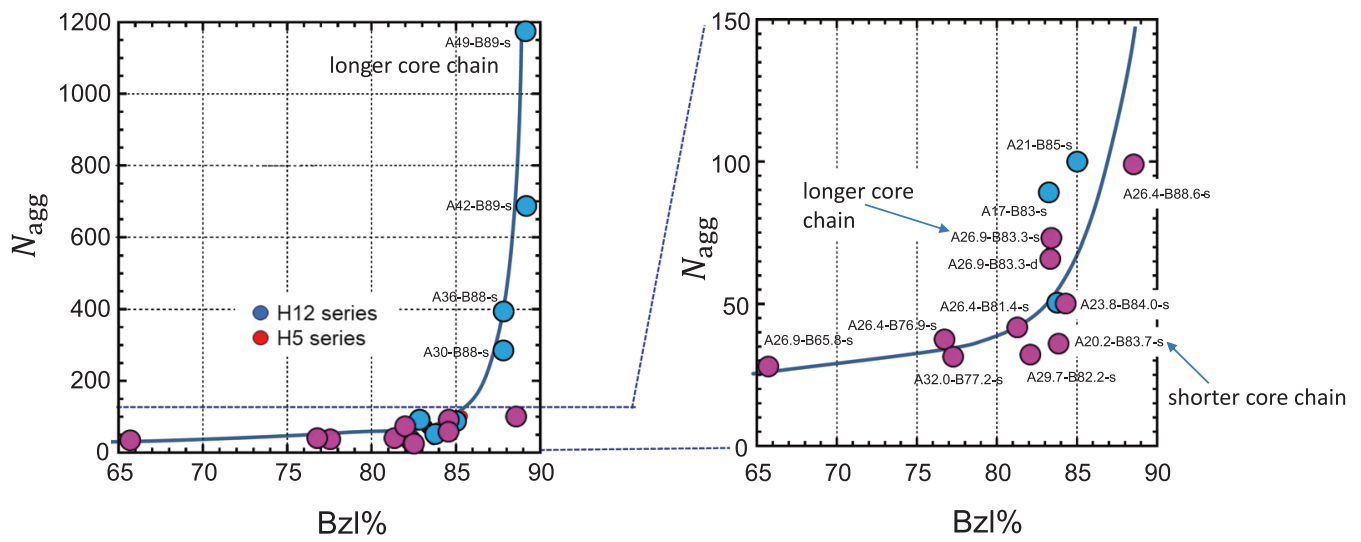

Fig. 17 The aggregation number $N_{\text {agg }}$ plotted against the benzylation ratio Bzl\% of the core chain

Fig. 18 The core chain length dependence of the aggregation number (A) and the relation of $N_{\text {agg }}$ and the ratio of $R_{\mathrm{C}}^{3} / L_{C}$
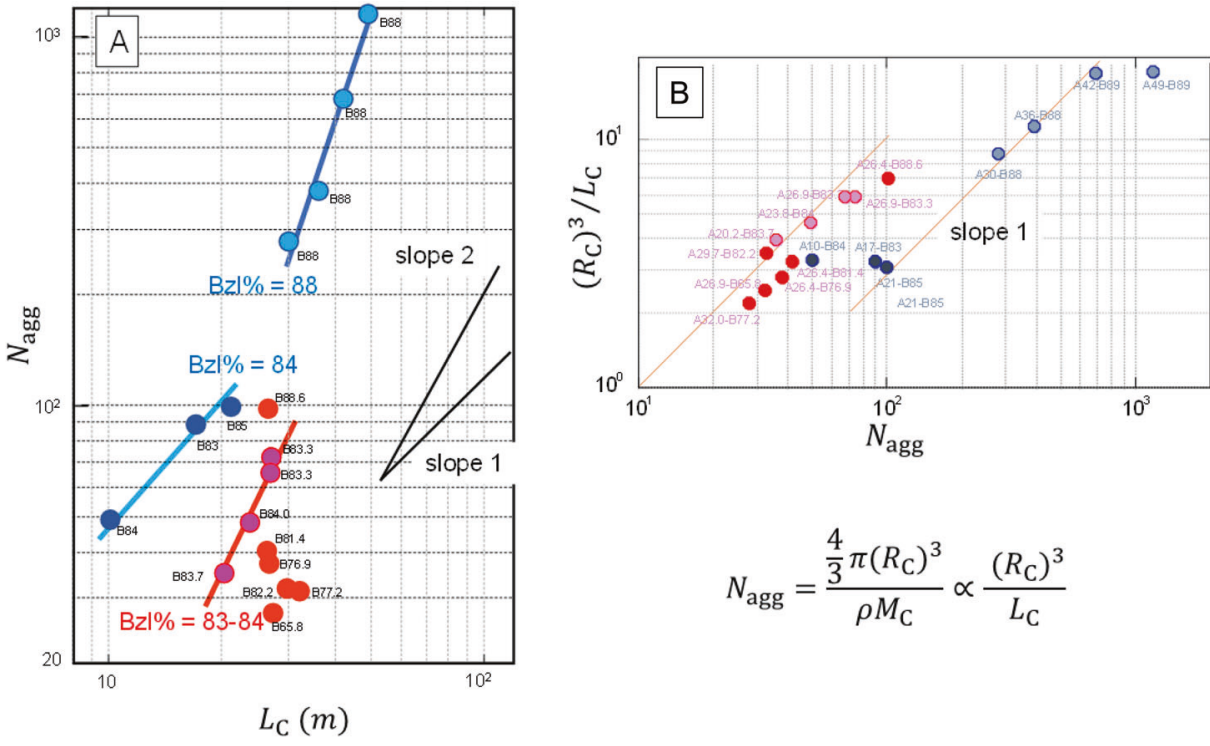

$N_{\text {agg }}=\frac{\frac{4}{3} \pi\left(R_{\mathrm{C}}\right)^{3}}{\rho M_{\mathrm{C}}} \propto \frac{\left(R_{\mathrm{C}}\right)^{3}}{L_{\mathrm{C}}}$ scattering measurements coupled with fractionation such as $\mathrm{FFF}+\mathrm{SLS}$ and GPC + SLS have great advantages over conventional batch measurements in terms of the regulatory science.

\section{The critical quality attributes (CQAs) to determine the micellar structures}

Figure 17 plots $N_{\text {agg }}$ against $\mathrm{Bzl} \%$ which appears to be the major factor in determining aggregation. When the carboxyl group was benzyl-esterified (see Fig. 13), this moiety became quite hydrophobic, and in reverse, the hydrated carboxyl group was hydrophilic. Therefore, $\mathrm{Bzl} \%$ is considered to reflect the hydrophobic/hydrophilic balance of the core $\mathrm{P}(\mathrm{Asp}(\mathrm{Bzl}))$ block. Therefore, the degree of hydrophobic interactions is the most significant factor to determine $N_{\text {agg }}$. In this plot, when comparing $N_{\text {agg }}$ at the same $\mathrm{Bzl} \%$, the longer core chain gives the larger $N_{\mathrm{agg}}$, indicating that the core chain length is the second major factor in determining $N_{\text {agg }}$. Figure 18A plots $N_{\text {agg }}$ against the core chain length $L_{C}$, indicating that the relation of $N_{\text {agg }} \propto L_{C}{ }^{\beta}$ may be held as long as we compare the samples that have the same Bzl\% and PEG chain length. However, the value of $\beta$ is much larger than expected from the predictions mentioned in Eqs. 1, 3, and 4. Figure $18 \mathrm{~B}$ plots $R_{\mathrm{C}}^{3} / L_{\mathrm{C}}$ against $N_{\text {agg }}$. Here we constructed the plot, considering that the relation of $\rho_{\text {core }} 4 / 3 \pi R_{\mathrm{C}}^{3}=N_{\mathrm{agg}} m L_{\mathrm{C}}$ should be held, where $\rho_{\text {core }}$ is the segment density of the core and $m$ is the mass of the repeating unit of the core chain. Within the similar Bzl\% and PEG chain length, the relation of $R_{\mathrm{C}}^{3} / L_{\mathrm{C}} \propto N_{\mathrm{agg}}$ is appeared to be held, but they do not lie on the same line when the PEG chain has a different length. This means that $\rho_{\text {core }}$ depends on the PEG chain length and Bzl\%. Furthermore, the slope of the data points are not exactly 1 . These phenome are not taken into account in the theory described in the section 2 , although note that there are many other systems consistent with the theoretical predictions [26-29, 37]. 

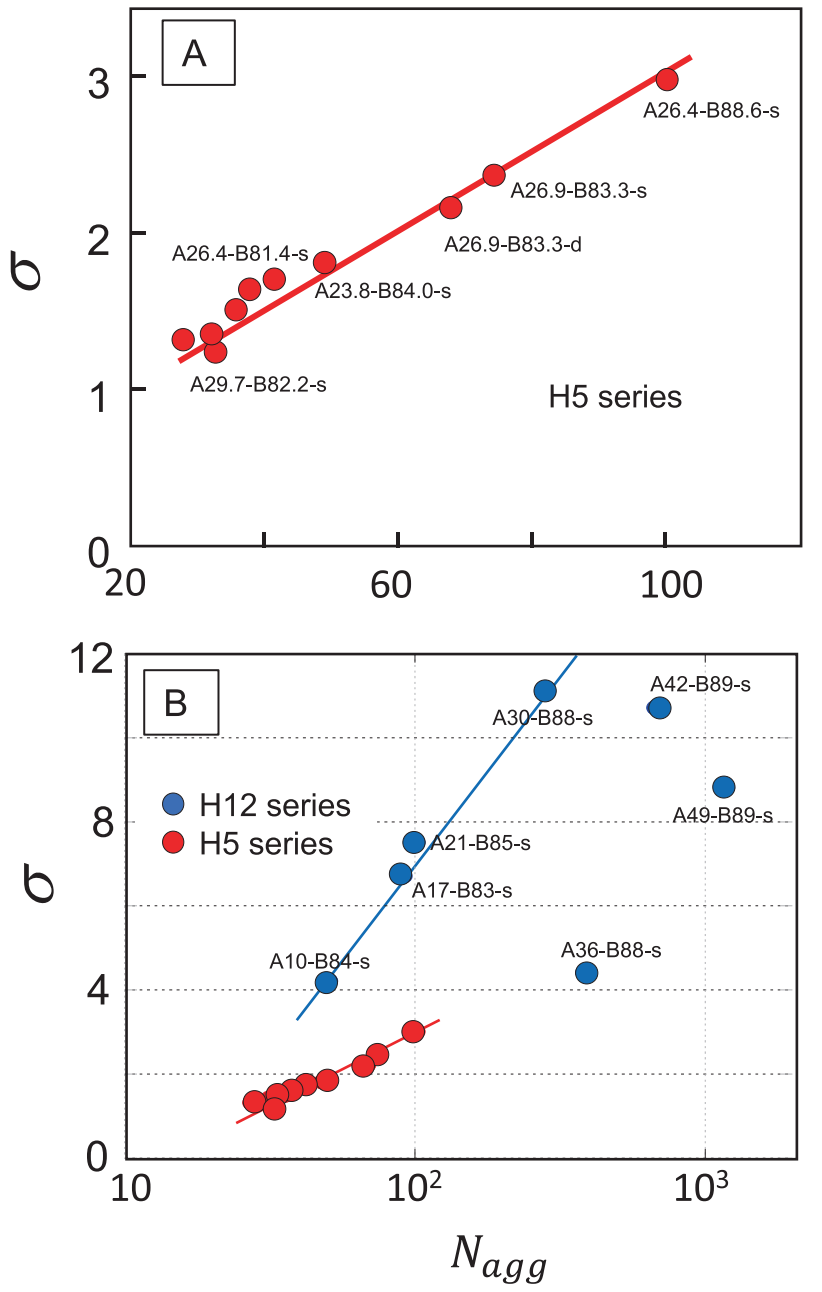

Fig. 19 The index of the PEG shell chain crowding $\sigma$ is plotted against the aggregation number $N_{\text {agg }}$ for only $\mathrm{H} 5$ series in $\mathbf{A}$ and all the samples in $\mathbf{B}$

In Fig. 19, the index $\sigma$ (defined by Eq. 2) to show how the shell PEG chains are crowding is plotted against $N_{\mathrm{agg}}$ for only the $\mathrm{H} 5$ series and in A and both in B. For H5, showing that $\sigma$ increases with an increase in $N_{\text {agg. }}$. Within the $\mathrm{H}$ series, there is a nice correlation between these two parameters, while the H12 series did not. Except for the larger core chain, the four points may be fitted by one straight line as shown in B. As shown in Fig. 16, $N_{\text {agg }}$ is closely related to the hydrophobicity of the core chain. Therefore, the hydrophobicity of the core chain may be the major factor in determining the PEG chain crowding.

\section{CQAs to determine the micellar stability in vitro and in vivo}

We found that $\sigma$ is closely related to the micellar stability, which is evaluated by determining how many fractions of the injected micelles are not trapped by a hydrophobic column (as illustrated in the schematic inset of Fig. 20). Yokoyama et al. reported that this stability was related to

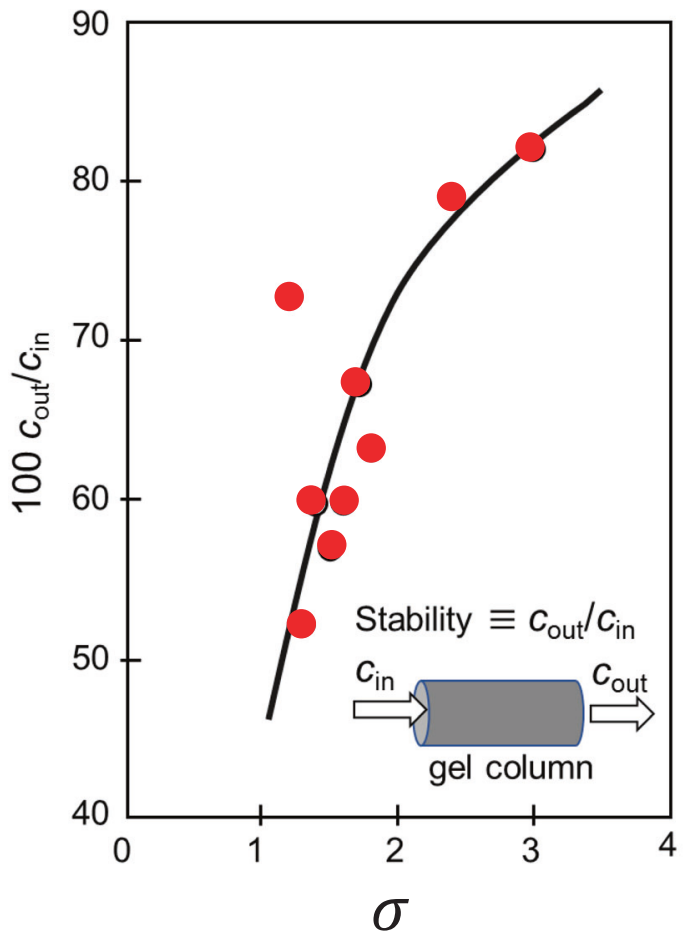

Fig. 20 The micellar physical stability parameters $\left(c_{\text {out }} / c_{\text {in }}\right)$ determined by recovery ratio of samples after passing a gel permeation chromatography column are plotted against $\sigma$

the initial burst of PEG-P[Asp(Bzl)] micelles when they were injected into mouse blood. The immediate and substantial micellar burst just after injection may cause an abrupt release of the drug and thus may spike the drug concentration up to a toxic level. Therefore, it is better to reduce the initial burst. The initial burst may be caused by a jump in osmotic pressure and unfavorable interaction with serum proteins. From the strong relationship between $\sigma$ and stability, we can conclude that greater PEG coverage of the hydrophobic core leads to a lower probability of interactions between serum proteins and the core, which might cause a reduction in the initial burst.

After carrying out the blood circulation test for four particles (H12-A49-B89-s, H5-A26-B89-s, H12-A10-B84-s, and H5-A26-B66-s) [68], we plotted the AUC (micellar concentration in blood area under the curve in vivo) against $\sigma$ (Fig. 21A). Here, a larger AUC means a longer blood circulation. By the way, there is another parameter $\left(D_{\mathrm{OS}}\right)$ to characterize the PEG crowding, especially focusing on the shell-water interface, which is given by:

$D_{\mathrm{OS}}=\frac{N_{\mathrm{agg}}}{4 \pi R_{S}^{2}}$

Figure 20B plots AUC against $D_{\text {OS }}$, showing that a higher density of PEG chains on the surface gives a higher 
Fig. 21 Schematic representations of the outermost shell surface's PEG density $\left(D_{\mathrm{OS}}\right)$ and the relationship between $\sigma$ and AUC in (A) and between $D_{\mathrm{OS}}$ and AUC in (B)
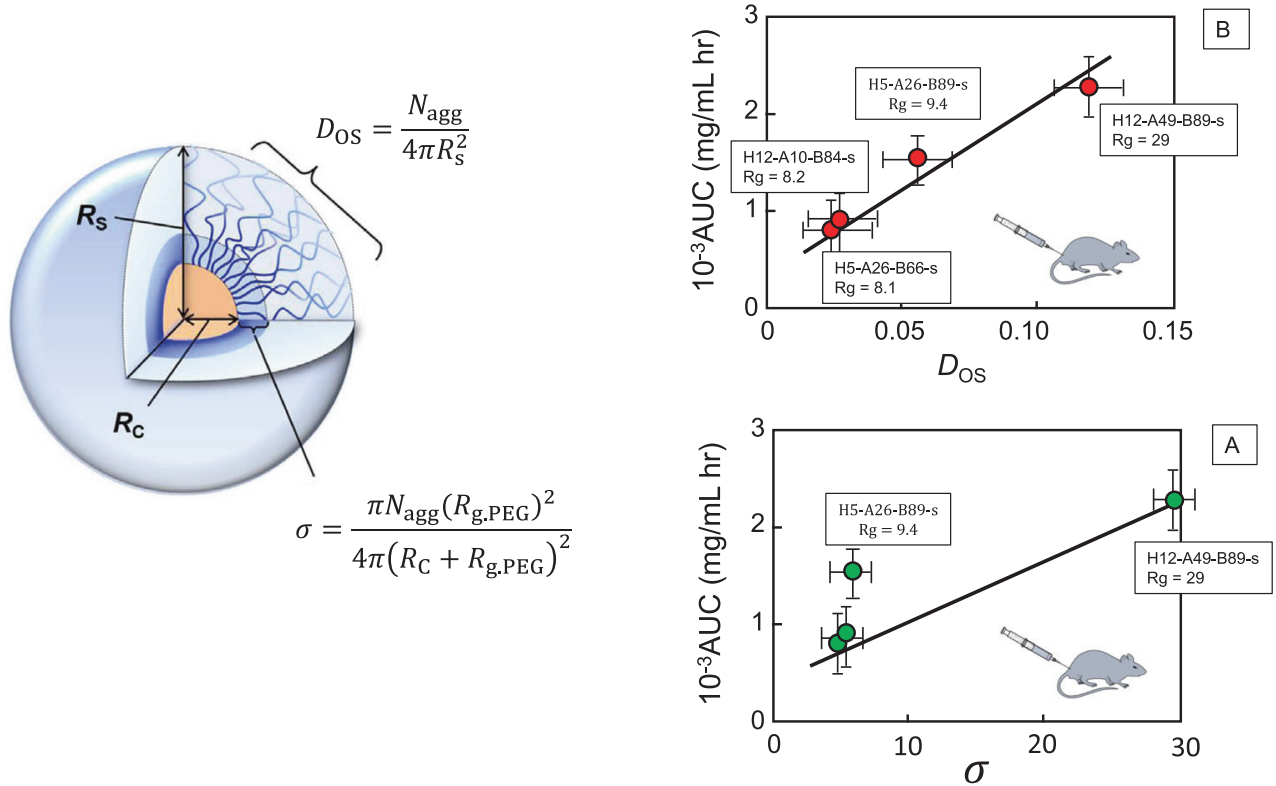

Fig. 22 Comparison of the scattering data of PEG-blockPBA and the PG model calculated with the parameters indicated in the illustration
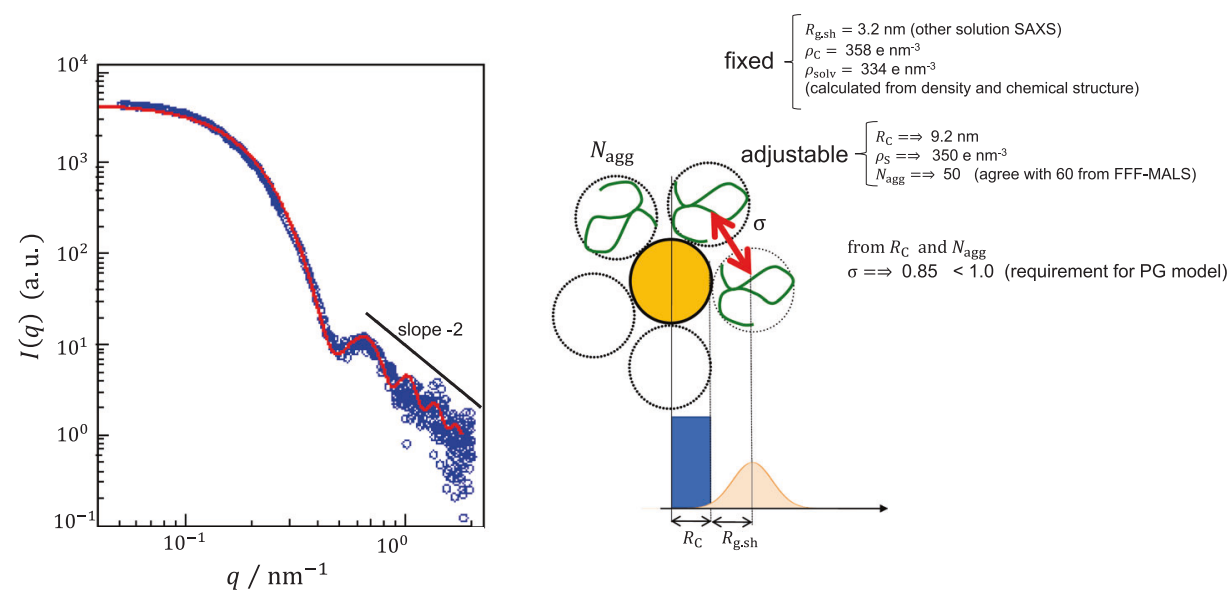

AUC. The correlation between AUC and $D_{\mathrm{OS}}$ is better than that of $\sigma$. This tendency can be interpreted as follows: the micelle surface is the first portion to contact serum proteins, and the larger PEG crowding can prevent the proteins from invading into the micelles and binding between the core and the proteins. $D_{\mathrm{OS}}$ and $\sigma$ are positively correlated with each other, although we found that AUC is more strongly correlated with $D_{\text {OS }}$ than $\sigma$. Therefore, the invaded proteins are also prevented from undergoing unfavorable interactions with the hydrophobic core. PEG nanoparticles are considered to accumulate in solid tumors by the EPR effect. This is the major mechanism by which anti-cancer drugs are selectively delivered to tumors. The longer blood circulation accelerates the accumulation of the drug in cancer cells. This means that $D_{\mathrm{OS}}$ is one of the important factors in controlling the efficacy of polymeric drugs.

\section{Application of the Pedersen-Gerstenberg (PG) model}

Poly(ethylene glycol)-block-poly(n-butyl acrylate), denoted by PEG-block-PBA, is an amphiphilic block copolymer that can be a standard for studies of polymer micelles and is easily synthesized. Here, we synthesized the block copolymer consisting of 220-mer of PEG and 97-mer of PBA (PEG220-block-PBA97) and prepared the micelles in aqueous solution. Figure 22 shows the SAXS profile of PEG220-block-PBA97 micelles in $1.0 \mathrm{mg} / \mathrm{mL}$ aqueous solution. In the low- $q$ region, $I(q)$ follows the Guinier law indicating that the micelles are isolated without the formation of secondary aggregation. The SAXS profile decays as $I(q) \propto q^{-2}$ in the high- $q$ region $\left(q>0.6 \mathrm{~nm}^{-1}\right)$, relating to scattering from the individual shell chains. This also suggests the tethered PEG chains are isolated enough with each 
Fig. 23 SAXS profile changes for the PEG-P(Asp(Bzl)) micelle upon LE540 loading. The inset magnifies the low- $q$ region (A) and magnified linear-linear plot in the high $q$ range to show the presence of ordering due to hexagonal chains of $\mathrm{P}(\mathrm{Asp}(\mathrm{Bzl}))$ and the disappearance upon addition of LE540 (B)

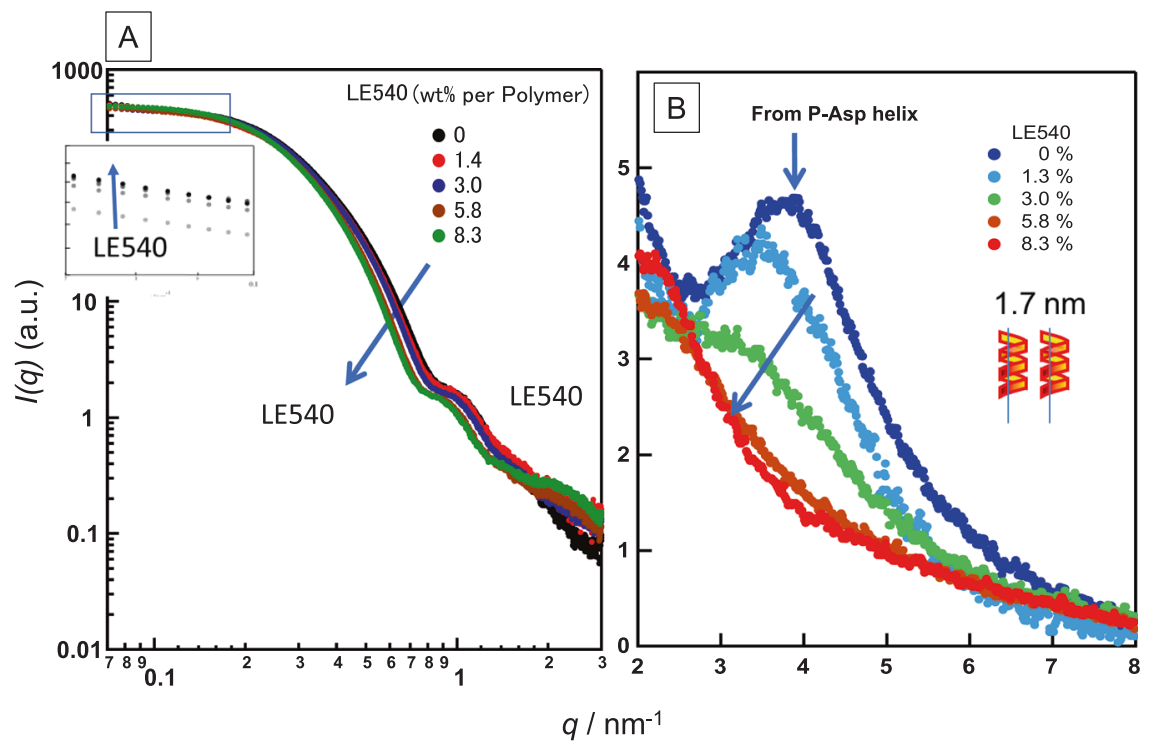

other (probably in the (a) state in Fig. 2). Thus, the SAXS profile was fitted with the PG model (Eq. 24). The solid line is the fitting result calculated with the fitting parameters for which are listed in the same figure. In the fitting analysis, the electron densities of core $\rho_{\mathrm{C}}$ and solvent $\rho_{\text {solv }}$ were fixed at 358 and $334 \mathrm{e} \mathrm{nm}^{-3}$, respectively, as evaluated by combining the densities $\left(1.09 \mathrm{~g} \mathrm{~cm}^{-3}\right.$ for PBA and $1.00 \mathrm{~g} \mathrm{~cm}^{-3}$ for $\mathrm{H}_{2} \mathrm{O}$ ) and chemical structures. Furthermore, $R_{\mathrm{g}}$ of the PEG homopolymer obtained from another SAXS measurement was used. Thus, the fitting analysis was performed with three adjustable parameters: the radius of the hydrophobic core $R_{\mathrm{C}}$, the electron density of the corona $\rho_{\mathrm{S}}$, and the aggregation number $N_{\text {agg. }}$. The calculated result is in good agreement with the experimental SAXS profile. The $R_{\mathrm{C}}(9.1 \mathrm{~nm})$ and $\rho_{\mathrm{S}}\left(350 \mathrm{e} \mathrm{nm}^{-3}\right)$ are reasonable in terms of the $R_{\mathrm{g}}$ value of PBA97 and density of PEG. $N_{\mathrm{agg}}$ does not much differ from the value obtained by FFF-MALS ( 60). Therefore, the PG model seems to explain the SAXS profiles of PEG220-block-PBA97 micelles well. When the $q^{-2}$ dependence of $I(q)$ in the high-q region was observed, the PG model is the first choice. In this case all the adjustable parameters used in the fitting should be consistent with other measurements especially $N_{\text {agg }}$ from light scattering.

\section{How are drug molecules incorporated into the core of polymeric micelles? [71]}

LE540 is a very hydrophobic retinoid antagonist drug (see Fig. 13B for the chemical structure); thus, we used LE540 as a model drug to study how PEG-P(Asp(Bzl)) micellar structures change with addition of LE540. We used H5A24-B83-s for this purpose. PEG-P(Asp(Bzl)) and LE540 were dissolved in THF at a desired loading ratio $\left(L_{\mathrm{LE} 540}\right)$, and then THF was evaporated at $45^{\circ} \mathrm{C}$ under $\mathrm{N}_{2}$ flow. The
PEG-P(Asp(Bzl))/LE540 mixtures were further dried under reduced pressure and then dispersed in $1 \times$ Dulbecco buffer at $25 \mathrm{mg} / \mathrm{mL}$, and the solution was ultrasonicated for a few minutes. The solutions were diluted to the desired concentration with the buffer, and the concentration of the micelle including LE540 was denoted by $C_{\mathrm{M}}$.

Figure 23A plots $I(q)$ against $q$ in the range of $0.07 \mathrm{~nm}^{-1}<$ $q<3 \mathrm{~nm}^{-1}$ for PEG-P(Asp(Bzl)) micelles at $L_{\mathrm{LE} 540}=0,1.4$, $3.0,5.8$, and $8.3 \mathrm{wt} \%$, and the profiles for $0.07<q<$ $0.1 \mathrm{~nm}^{-1}$ are magnified in the inset. The $q$ range in the figures should reflect the micelle size and its inner structures. All SAXS profiles converge to a plateau at a smaller $q$, confirming that PEG- $\mathrm{P}(\mathrm{Asp}(\mathrm{Bzl}))$ and the drug-loaded ones indeed formed an isolated micelle. The profiles showed the secondary maximum at $q \approx 0.9 \mathrm{~nm}^{-1}$, suggesting that the micelles had a well-defined structure with a relatively narrow size distribution. There was a minimum or shoulder around $q=0.7-0.8 \mathrm{~nm}^{-1}$, indicating that the radius of the particle is $\sim 6.1-5.6 \mathrm{~nm}$ as estimated from the relation $R_{\mathrm{C}} q^{*}=4.493$ for the solid sphere (see Fig. 4). With increasing $L_{\mathrm{LE} 540}$, the SAXS profiles were shifted to lower $q$. This feature can be interpreted as the expansion of micelle size upon incorporation of LE540, presumably into the core. The absolute values of $I(q)$ at lower $q$ increased with increasing LLE540.

Figure 23B shows the SAXS profiles at $2 \mathrm{~nm}^{-1}<q<$ $8 \mathrm{~nm}^{-1}$, corresponding to $0.4-3 \mathrm{~nm}$ in real space. The micelles at $L_{\mathrm{LE} 540}=0$ and $1.4 \mathrm{wt} \%$ exhibited a clear diffraction peak at $q \approx 4 \mathrm{~nm}^{-1}$. According to the bulk poly ( $\beta$-benzyl-1-aspartate) (PBLA) data [74], this peak can be attributed to ordering between the helices made from $\mathrm{P}$ (Asp (Bzl)) chains, called a nonspecific hexagonal arrangement. The diffraction peak becomes drastically weaker with increasing $L_{\mathrm{LE5} 40}$ and eventually disappears. The decrease in the peak 

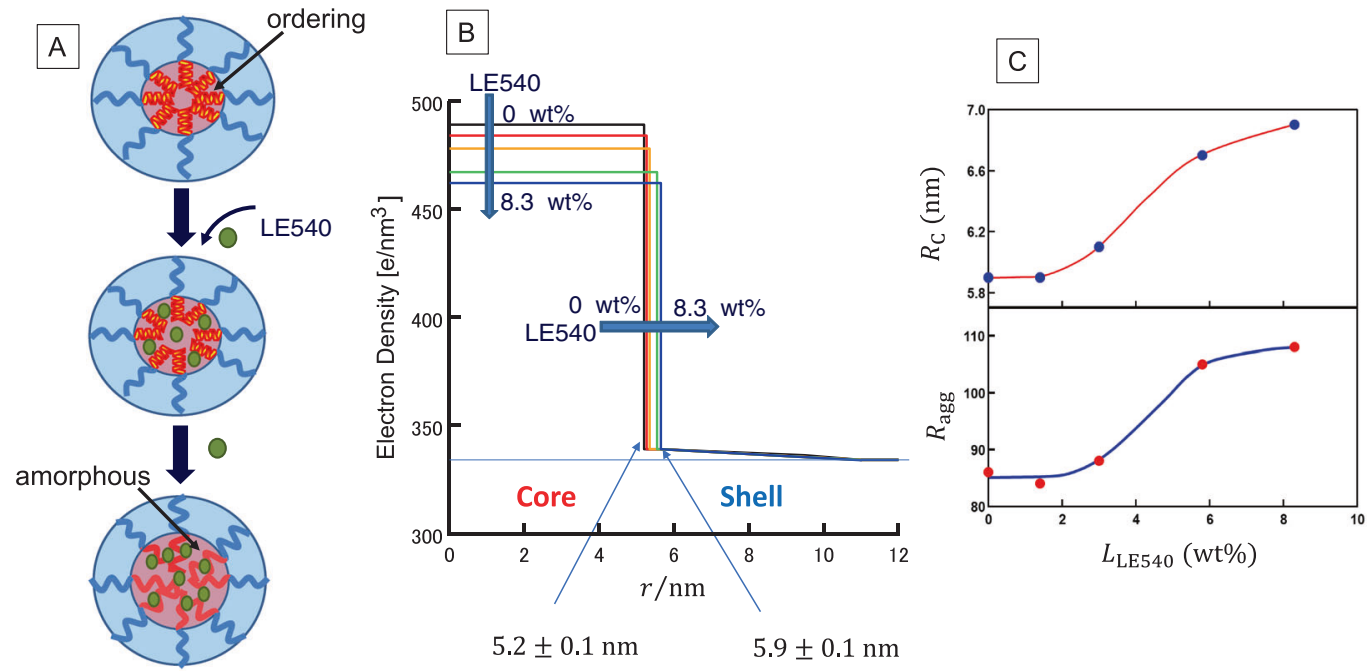

Fig. 24 The schematic illustrations to show how the micellar structures are changed upon loading of LE540 (A, B). The LE540 loading ratio dependence of $R_{\mathrm{C}}$ and $N_{\mathrm{agg}}(\mathbf{C})$

intensity corresponds to a decrease in the ordering of the $\mathrm{P}(\mathrm{Asp}(\mathrm{Bzl}))$ helices. This phenomenon can be interpreted as a uniform distribution of LE540 in the P(Asp(Bzl)) core, and the interaction between LE540 and $\mathrm{P}(\mathrm{Asp}(\mathrm{Bzl}))$ is more favorable than the helix formation of $\mathrm{P}(\mathrm{Asp}(\mathrm{Bzl}))$.

Based on the above data, we can visualize how the addition of LE540 changes the micellar structures as illustrated in Fig. 24. The peak ascribed to the crystalline-like ordering disappeared as LE540 was immersed in the core. This result can be interpreted by assuming that LE540 was uniformly distributed in the core and decreasing the preexisting ordering of Asp(Bzl) chains, as illustrated in Fig. 24A. Upon the addition of LE540, the core density decreased and the core size increased, while the shell size appeared to remain the same, as shown in B. The aggregation number and the core size increased sigmoidally, as shown in $\mathrm{C}$.

\section{Concluding remarks}

Throughout this review paper, we have made clear that the accurate structural analysis of polymeric micelles in solutions can be carried out by using static-scattering techniques, especially SAXS. SAXS has a great advantage in examining the structures whose sizes are less than $100 \mathrm{~nm}$. This size range is exactly that of the structure from which the function of nanomedicines is derived. In terms of the regulatory science of drugs, many CQAs for the safety and efficacy of nanomedicines should be related to their structures and properties; therefore, SAXS can be a key technique.
Acknowledgements This work is financially supported by the JST CREST program and all SAXS measurements were carried out at SPring-8 40B2 (2008B0012, 2008B1781, 2009A0012, 2009B1397, 2010A1089, 2010B1726, 2011A1668, 2011B1735). We appreciate for the long-term collaboration with Profs. Yuya Shinohara, Yoshiyuki Amemiya, and Naoto Yagi through scattering experiments and theoretical discussion during the JST CREST program. All PEG-P(Asp (Bzl)) samples were synthesized by Profs Kouichi Shiraishi and Masayuki Yokoyama and we would like to thank them; without their samples and knowledge of the system, we would not have had anything done. The in vivo experiment was carried out by Prof Kumi Kawano. The authors thank all the laboratory members at the University of Kitakyushu involved in this project, especially Drs. Yusuke Sanada and Rintaro Takahashi. Finally, KS would like to thank his mentors who have educated and guided him in the field of macromolecular science: Profs. Hiroshi Fujita, Akio Teramoto, Takashi Norisuye, Willan J. MacKnight, and Seiji Shinkai. KS would not have won the 2017 SPSJ award without their guidance.

\section{Compliance with ethical standards}

Conflict of interest The authors declare no competing interests.

Publisher's note Springer Nature remains neutral with regard to jurisdictional claims in published maps and institutional affiliations.

Open Access This article is licensed under a Creative Commons Attribution 4.0 International License, which permits use, sharing, adaptation, distribution and reproduction in any medium or format, as long as you give appropriate credit to the original author(s) and the source, provide a link to the Creative Commons license, and indicate if changes were made. The images or other third party material in this article are included in the article's Creative Commons license, unless indicated otherwise in a credit line to the material. If material is not included in the article's Creative Commons license and your intended use is not permitted by statutory regulation or exceeds the permitted use, you will need to obtain permission directly from the copyright holder. To view a copy of this license, visit http://creativecommons. org/licenses/by/4.0/. 


\section{References}

1. Mai Y, Eisenberg A. Self-assembly of block copolymers. Chemical Soc Rev. 2012;41:5969-85.

2. Zhang L, Eisenberg A. Multiple morphologies of "crew-cut" aggregates of polystyrene-b-poly (acrylic acid) block copolymers. Science. 1995;268:1728-31.

3. Hayward RC, Pochan DJ. Tailored assemblies of block copolymers in solution: it is all about the process. Macromolecules. 2010;43:3577-84. https://doi.org/10.1021/ma9026806.

4. Zhulina EB, Borisov OV. Theory of block polymer micelles: recent advances and current challenges. Macromolecules. 2012;45:4429-40. https://doi.org/10.1021/ma300195n.

5. Hamley IW. The Physics of Block Copolymers. New York: Oxford University Press; 1998.

6. Tuzar Z, Kratochvíl P. Block and graft copolymer micelles in solution. Adv Coll Interface Sci. 1976;6:201-32. https://doi.org/ 10.1016/0001-8686(76)80009-7.

7. Hamley IW. Block copolymers in solution: fundamentals and applications. West Sussex, England: John Wiley \& Sons; 2005. https://onlinelibrary.wiley.com/doi/book/10.1002/9780470016985.

8. Lu Y, Park K. Polymeric micelles and alternative nanonized delivery vehicles for poorly soluble drugs. Int $\mathrm{J}$ Pharm. 2013;453:198-214.

9. Kataoka K, Harada A, Nagasaki Y. Block copolymer micelles for drug delivery: design, characterization and biological significance. Adv Drug Deliv Rev. 2001;47:113-31. https://doi.org/10.1016/ s0169-409x(00)00124-1.

10. Cabral H, Miyata K, Osada K, Kataoka K. Block copolymer micelles in nanomedicine applications. Chem Rev. 2018;118:6844-92. https://doi.org/10.1021/acs.chemrev.8b00199.

11. Yokoyama M. Clinical applications of polymeric micelle carrier systems in chemotherapy and image diagnosis of solid tumors. $\mathrm{J}$ Exp Clin Med. 2011;3:151-8. https://doi.org/10.1016/j.jecm. 2011.06.002.

12. Miyata K, Nishiyama N, Kataoka K. Rational design of smart supramolecular assemblies for gene delivery: chemical challenges in the creation of artificial viruses. Chem Soc Rev. 2012;41:2562-74. https://doi.org/10.1039/C1CS15258K.

13. Gref R, Lück M, Quellec P, Marchand M, Dellacherie E, Harnisch S, Blunk T, Müller RH. 'Stealth' corona-core nanoparticles surface modified by polyethylene glycol (PEG): influences of the corona (PEG chain length and surface density) and of the core composition on phagocytic uptake and plasma protein adsorption. Coll Surf B. 2000;18:301-13. https://doi.org/10.1016/S0927-7765 (99)00156-3.

14. Cabral H, Matsumoto Y, Mizuno K, Chen Q, Murakami M, Kimura M, Terada Y, Kano MR, Miyazono K, Uesaka M, Nishiyama N, Kataoka K. Accumulation of sub-100 nm polymeric micelles in poorly permeable tumours depends on size. Nat Nanotechnol. 2011;6:815-23. https://doi.org/10.1038/nnano. 2011.166.

15. Maeda H, Wu J, Sawa T, Matsumura Y, Hori K. Tumor vascular permeability and the EPR effect in macromolecular therapeutics: a review. J Control Release. 2000;65:271-84.

16. Maeda H, Tsukigawa K, Fang J. A retrospective 30 years after discovery of the enhanced permeability and retention effect of solid tumors: next-generation chemotherapeutics and photodynamic therapy-problems, solutions, and prospects. Microcirculation. 2016;23:173-82. https://doi.org/10.1111/micc.12228.

17. Min Y, Caster JM, Eblan MJ, Wang AZ. Clinical translation of nanomedicine. Chem Rev. 2015;115:11147-90. https://doi.org/ 10.1021/acs.chemrev.5b00116.

18. Israelachvili JN. Intermolecular and surface forces. Vol. 450. London: Academic press London; 1992.
19. Israelachvili JN, Mitchell DJ, Ninham BW. Theory of selfassembly of hydrocarbon amphiphiles into micelles and bilayers. $\mathrm{J}$ Chem Soc Faraday Trans 2. 1976;72:1525.

20. De Gennes PG. In Liquid Crystals (Solid state physics: Supplement; 14) (Liebert, L. ed). Academic Press Inc; 1978. pp. 1-18.

21. de Gennes PG. Conformations of polymers attached to an interface. Macromolecules. 1980;13:1069-75. https://doi.org/10.1021/ ma60077a009.

22. Svaneborg C, Pedersen JS. Form factors of block copolymer micelles with excluded-volume interactions of the corona chains determined by Monte Carlo simulations. Macromolecules. 2002;35:1028-37. https://doi.org/10.1021/ma011046v.

23. Halperin A. Polymeric micelles: a star model. Macromolecules. 1987;20:2943-6. https://doi.org/10.1021/ma00177a051.

24. Zhulina YB, Birshtein TM. Conformations of block-copolymer molecules in selective solvents (micellar structures). Poly Scie U. S.S.R. 1985;27:570-8, https://doi.org/10.1016/0032-3950(85) 90238-2.

25. Daoud D, Cotton JP. Star shaped polymers:a model for the conformation and its concentration dependence. $J$ Phys. 1982;43:531-8.

26. Nagarajan R, Ganesh K. Solubilization in spherical block copolymer micelles: scaling analysis based on star model. J Chem Phys. 1993;98:7440-50.

27. Nagarajan R, Ganesh K. Block copolymer self-assembly in selective solvents: spherical micelles with segregated cores. J Chem Phys. 1989;90:5843-56. https://doi.org/10.1063/1.456390.

28. Nagarajan R, Ganesh K. Block copolymer self-assembly in selective solvents: theory of solubilization in spherical micelles. Macromolecules. 1989;22:4312-25.

29. Nagarajan R. Constructing a molecular theory of self-assembly: Interplay of ideas from surfactants and block copolymers. Adv Coll Interface Sci. 2017;244:113-23. https://doi.org/10.1016/j.cis. 2016.12.001.

30. Antonietti M, Heinz S, Schmidt M, Rosenauer CJM. Determination of the micelle architecture of polystyrene/poly (4-vinylpyridine) block copolymers in dilute solution. Macromolecules. 1994;27:3276-81.

31. Chen F, Wu D. Theoretical predictions of free energy and aggregation number for star-shaped micelles. Acta Poly Sin. 2000;385. https://jglobal.jst.go.jp/en/detail?JGLOBAL_ID= 200902147600284170.

32. Ma Y, Lodge TP. Poly(methyl methacrylate)-block-poly(n-butyl methacrylate) diblock copolymer micelles in an ionic liquid: scaling of core and corona size with core block length. Macromolecules. 2016;49:3639-46. https://doi.org/10.1021/acs.ma cromol.6b00315.

33. Mössmer S, Spatz JP, Möller M, Aberle T, Schmidt J, Burchard W. Solution behavior of poly(styrene)-\&Zoc\&-poly(2-vinylpyridine) micelles containing gold nanoparticles. Macromolecules. 2000;33:4791-8. https://doi.org/10.1021/ma992006i.

34. Zhang L, Eisenberg A. Structures of "crew-cut" aggregates of polystyrene-b-poly(acrylic acid) diblock copolymers. Macromol Symp. 1997;113:221-32. https://doi.org/10.1002/masy. 19971130119.

35. Burguière C, Chassenieux C, Charleux B. Characterization of aqueous micellar solutions of amphiphilic block copolymers of poly(acrylic acid) and polystyrene prepared via ATRP. Toward the control of the number of particles in emulsion polymerization. Polymer. 2002;44:509-18. https://doi.org/10.1016/S0032-3861 (02)00811-X.

36. Förster S, Zisenis M, Wenz E, Antonietti M. Micellization of strongly segregated block copolymers. J Chem Phys. 1996;104:9956-70. https://doi.org/10.1063/1.471723.

37. Takahashi R, Miwa S, Sobotta FH, Lee JH, Fujii S, Ohta N, Brendel JC, Sakurai K. Unraveling the kinetics of the structural 
development during polymerization-induced self-assembly: Decoupling the polymerization and the micelle structure. Polym. Chem.2020;11:1514-24. https://doi.org/10.1039/c9py01810g.

38. Ng R. Drugs: from discovery to approval. New Jersey: John Wiley \& Sons; 2015. https://www.wiley.com/en-us/Drugs\%3A+From +Discovery+to+Approval\%2C+3rd+Edition-p-9781118907276.

39. D'Mello SR, Cruz CN, Chen M-L, Kapoor M, Lee SL, Tyner KM. The evolving landscape of drug products containing nanomaterials in the United States. Nat Nanotechnol. 2017;12:523 https://doi.org/10.1038/nnano.2017.67.

40. FDA. U.S. FDA. Draft guidance for industry: drug products, including biological products, that contain nanomaterials. 2017. https://www.fda.gov/downloads/Drugs/GuidanceComplia nceRegulatoryInformation/Guidances/UCM588857.pdf.

41. Coty J-B, Vauthier C. Characterization of nanomedicines: A reflection on a field under construction needed for clinical translation success. J Control Release. 2018;275:254-68. https://doi. org/10.1016/j.jconrel.2018.02.013.

42. Gioria S, Caputo F, Urbán P, Maguire CM, Bremer-Hoffmann S, Prina-Mello A, Calzolai L, Mehn D. Are existing standard methods suitable for the evaluation of nanomedicines: Some case studies. Nanomedicine. 2018;13:539-54. https://doi.org/10.2217/ nnm-2017-0338.

43. Caputo F, Clogston J, Calzolai L, Roesslein M, Prina-Mello A. Measuring particle size distribution of nanoparticle enabled medicinal products, the joint view of EUNCL and NCI-NCL. A step by step approach combining orthogonal measurements with increasing complexity. J Control Release. 2019;299: 31-43.

44. Hafner A, Lovrić J, Lakoš GP, Pepić I. Nanotherapeutics in the EU: an overview on current state and future directions. Int $\mathbf{J}$ Nanomed. 2014;9:1005-23. https://doi.org/10.2147/IJN.S55359.

45. Use, C. f. M. P. f. H. Joint MHLW/EMA reflection paper on the development of block copolymer micelle medicinal products. Eur Med Age Lond. 2013;19. https://www.ema.europa.eu/ development-block-copolymer-micelle-medicinal-products.

46. FDA. Liposome Drug Products: Chemistry, Manufacturing, and Controls; Human Pharmacokinetics and Bioavailability; and Labeling Documentation. 2018. https://www.fda.gov/regulatoryinformation/search-fda-guidance-documents/liposome-drugproducts-chemistry-manufacturing-and-controls-human-pharma cokinetics-and.

47. Grabarek AD, Weinbuch D, Jiskoot W, Hawe A. Critical evaluation of microfluidic resistive pulse sensing for quantification and sizing of nanometer- and micrometer-sized particles in biopharmaceutical products. J Pharm Sci. 2019;108:563-73. https:// doi.org/10.1016/j.xphs.2018.08.020.

48. Pedersen JS. Analysis of small-angle scattering data from colloids and polymer solutions: modeling and least-squares fitting. Adv Coll Interface Sci. 1997;70:171-210.

49. Kotlarchyk M, Chen S-H. Analysis of small angle neutron scattering spectra from polydisperse interacting colloids. J Chem Phys. 1983;79:2461-9.

50. Naruse K, Eguchi K, Akiba I, Sakurai K, Masunaga H, Ogawa H, Fossey JS. Flexibility and cross-sectional structure of an anionic dual-surfactant wormlike micelle explored with smallangle X-ray scattering coupled with contrast variation technique. J Phys Chem B. 2009;113:10222-9. https://doi.org/10. 1021/jp9019415.

51. Nakano M, Deguchi M, Matsumoto K, Matsuoka H, Yamaoka H. Self-Assembly of Poly(1,1-diethylsilabutane)-block-poly(2hydroxyethyl methacrylate) Block Copolymer. 1. Micelle Formation and Micelle-Unimer-Reversed Micelle Transition by Solvent Composition. Macromolecules. 1999;32:7437-43. https:// doi.org/10.1021/ma981912c.
52. Pedersen JS, Gerstenberg MC. Scattering form factor of block copolymer micelles. Macromolecules. 1996;29:1363-5. https:// doi.org/10.1021/ma9512115.

53. Pedersen JS. Form factors of block copolymer micelles with spherical, ellipsoidal and cylindrical cores. J Appl Crystallogr. 2000;33:637-40. https://doi.org/10.1107/S0021889899012248.

54. Pedersen JS. Structure factors effects in small-angle scattering from block copolymer micelles and star polymers. J Chem Phys. 2001;114:2839-46.

55. Svaneborg C, Pedersen JS. Form factors of block copolymer micelles with excluded-volume interactions of the corona chains determined by Monte Carlo simulations. Macromolecules. 2001;35:1028-37. https://doi.org/10.1021/ma011046v.

56. Pedersen JS, Schurtenberger P. Scattering functions of semiflexible polymers with and without excluded volume effects. Macromolecules. 1996;29:7602-12. https://doi.org/10.1021/ma9607630.

57. Sanada Y, Matsuzaki T, Mochizuki S, Okobira T, Uezu K, Sakurai K. $\beta$-1,3-d-glucan schizophyllan/poly(dA) triple-helical complex in dilute solution. J Phys Chem B. 2012;116:87-94. https://doi.org/10.1021/jp209027u.

58. Takahashi R, Fujii S, Akiba I, Sakurai K. Scattering form factor of block copolymer micelles with corona chains discretely distributed on the core surface. J Phys Chem B. 2020;124:6140-6. https://doi.org/10.1021/acs.jpcb.0c04120.

59. Lee JH, Fujii S, Takahashi R, Sakurai K. Monodisperse micelles with aggregation numbers related to platonic solids. Macromol Rapid Commun. 2020;41:2000227. https://doi.org/10.1002/marc. 202000227.

60. Mylonas E, Yagi N, Fujii S, Ikesue K, Ueda T, Moriyama H, Sanada Y, Uezu K, Sakurai K, Okobira T. Structural analysis of a calix [4] arene-based Platonic Micelle. Sci Rep. 2019;9:1982.

61. Miyake R, Fujii S, Lee JH, Takahashi R, Sakurai K. Dual and multiple stimuli-responsive platonic micelles bearing disaccharides. J Coll Interface Sci. 2019;535:8-15.

62. Lee JH, Matsumoto H, Fujii S, Takahashi R, Sakurai K. Monodisperse micelles composed of poly (ethylene glycol) attached surfactants: Platonic nature in a macromolecular aggregate. Soft Matter. 2019;15:5371-4.

63. Yoshida K, Fujii S, Takahashi R, Matsumoto S, Sakurai K. SelfAssembly of Calix [4] arene-Based Amphiphiles Bearing Polyethylene Glycols: Another Example of "Platonic Micelles". Langmuir. 2017;33:9122-8.

64. Fujii S, Yamada S, Matsumoto S, Kubo G, Yoshida K, Tabata E, Miyake R, Sanada Y, Akiba I, Okobira T. Platonic micelles: monodisperse micelles with discrete aggregation numbers corresponding to regular polyhedra. Sci Rep. 2017;7:44494.

65. Clare BW, Kepert DL. The closest packing of equal circles on a sphere. Proc R Soc Lond A. 1986;405:329-44. https://doi.org/10. 1098/rspa.1986.0056.

66. Saff EB, Kuijlaars AB. Distributing many points on a sphere. Math Intell. 1997;19:5-11.

67. Erber $\mathrm{T}$, Hockney G. Equilibrium configurations of $\mathrm{N}$ equal charges on a sphere. J Phys A. 1991;24:L1369.

68. Shiraishi K, Sanada Y, Mochizuki S, Kawano K, Maitani Y, Sakurai K, Yokoyama M. Determination of polymeric micelles' structural characteristics, and effect of the characteristics on pharmacokinetic behaviors. J Control Release. 2015;203:77-84.

69. Sanada Y, Akiba I, Sakurai K, Shiraishi K, Yokoyama M, Mylonas E, Ohta N, Yagi N, Shinohara Y, Amemiya Y. Hydrophobic molecules infiltrating into the poly(ethylene glycol) domain of the core/shell interface of a polymeric micelle: evidence obtained with anomalous small-angle X-ray scattering. J Am Chem Soc. 2013;135:2574-82. https://doi.org/10.1021/ja308965j.

70. Sanada Y, Akiba I, Hashida S, Shiraishi K, Yokoyama M, Yagi N, Shinohara Y, Amemiya Y, Sakurai K. Characterization of 
polymer micelles by the combination of SAXS and FFF-MALS. Kobunshi Ronbunshu. 2012;69:346-57.

71. Akiba I, Terada N, Hashida S, Sakurai K, Sato T, Shiraishi K, Yokoyama M, Masunaga H, Ogawa H, Ito K. Encapsulation of a hydrophobic drug into a polymer-micelle core explored with synchrotron SAXS. Langmuir. 2010;26:7544-51.

72. Yamamoto T, Yokoyama M, Opanasopit P, Hayama A, Kawano $\mathrm{K}$, Maitani $\mathrm{Y}$. What are determining factors for stable drug incorporation into polymeric micelle carriers? Consideration on physical and chemical characters of the micelle inner core. J
Control Release. 2007;123:11-18. https://doi.org/10.1016/j. jconrel.2007.07.008.

73. Barz M, Luxenhofer R, Zentel R, Vicent MJ. Overcoming the PEG-addiction: well-defined alternatives to PEG, from structureproperty relationships to better defined therapeutics. Polym. Chem. 2011;2:1900-18. https://doi.org/10.1039/c0py00406e.

74. Tanaka S, Ogura A, Kaneko T, Murata Y, Akashi M. Precise synthesis of ABA triblock copolymers comprised of poly(ethylene oxide) and poly(-benzyl-1-aspartate): a hierarchical structure inducing excellent elasticity. Macromolecules. 2004;37:1370-7. https://doi.org/10.1021/ma035472n.

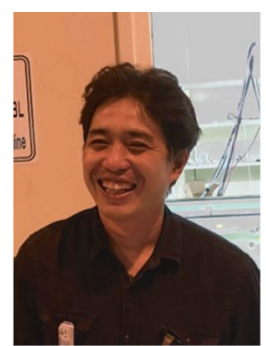

Isamu Akibais a professor of The University of Kitakyushu. He received his Ph.D. from Tokyo University of Agriculture and Technology (TUAT) in 2000. He worked as a researcher at Kuraray Co. Ltd. and then he became a research associate at TUAT. In 2001, he moved to The University of Kitakyushu (UKK) as a lecturer. After he worked as an associate professor, he has been in the current position at UKK from 2009. His research interests are in the self-assembly of amphiphilic molecules, such as block copolymers and surfactants, and the elucidation of hierarchical structures of soft materials by using quantum beams.

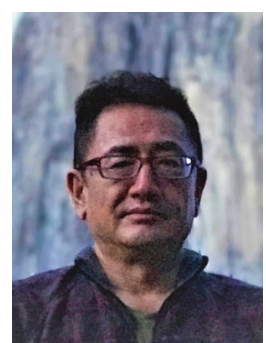

Kazuo Sakuraihas spent 16 years at the central research center of Kanebo Ltd. and has been involved in the fundamental research and business development of conductive polymers for batteries, optical polyester resin, and drug-delivering systems. He spent three years (1990-1993) in the US working for Prof. MacKnight at the University of Massachusetts, involved in polymer blends and X-ray and neutron scattering. Based on the works in the US, he received Ph.D. from Osaka University in 1996. After he came back to Japan, he was the leader of a research group to develop optical-lens polyesters, and their product has been used in most the cell-phones. He worked for Pro. Seiji Shinkai in a JST Project at Kurume from 1999-2001. He has been a professor at the University of Kitakyushu since 2001. He obtained several research funds from CREST/JST (twice), NeXTEP/JST, and the National Institute of Biomedical Innovation (NIBIO), which are well-known as Japan's most competitive funds. His primary research interests are polysaccharides, polysaccharide/polynucleotide complexes, gene delivery, and small-angle X-ray scattering from soft materials, especially from bio-related materials. $\mathrm{He}$ is one of the founders of a bio-venture company called NapaJen and Chief Scientific Officer. 